\title{
Advanced numerical studies of the neutralized drift compression of intense ion beam pulses
}

\author{
Adam B. Sefkow and Ronald C. Davidson \\ Plasma Physics Laboratory, Princeton University, Princeton, New Jersey 08543, USA
}

(Received 16 June 2007; published 24 October 2007)

\begin{abstract}
Longitudinal bunch compression of intense ion beams for warm dense matter and heavy ion fusion applications occurs by imposing an axial velocity tilt onto an ion beam across the acceleration gap of a linear induction accelerator, and subsequently allowing the beam to drift through plasma in order to neutralize its space-charge and current as the pulse compresses. The detailed physics and implications of acceleration gap effects and focusing aberration on optimum longitudinal compression are quantitatively reviewed using particle-in-cell simulations, showing their dependence on many system parameters. Finitesize gap effects are shown to result in compression reduction, due to an increase in the effective longitudinal temperature imparted to the beam, and a decrease in intended fractional tilt. Sensitivity of the focal plane quality to initial longitudinal beam temperature is explored, where slower particles are shown to experience increased levels of focusing aberration compared to faster particles. A plateau effect in axial compression is shown to occur for larger initial pulse lengths, where the increases in focusing aberration over the longer drift lengths involved dominate the increases in relative compression, indicating a trade-off between current compression and pulse duration. The dependence on intended fractional tilt is also discussed and agrees well with theory. A balance between longer initial pulse lengths and larger tilts is suggested, since both increase the current compression, but have opposite effects on the final pulse length, drift length, and amount of longitudinal focusing aberration. Quantitative examples are outlined that explore the sensitive dependence of compression on the initial kinetic energy and thermal distribution of the beam particles. Simultaneous transverse and longitudinal current density compression can be achieved in the laboratory using a strong final-focus solenoid, and simulations addressing the effects of focusing aberration in both directions are presented.
\end{abstract}

DOI: 10.1103/PhysRevSTAB.10.100101

PACS numbers: 52.59.Sa, 41.75.-i, 52.65.Rr, 29.27.-a

\section{INTRODUCTION}

Of the challenges encountered in developing heavy ion drivers [1] for warm dense matter and heavy ion fusion applications [2-4], one of the most significant is found in the final transport section leading to the target, where ion beam compression in space and time is required in order to achieve the necessary high intensities for striking the target $[5,6]$. In other words, intense ion beam pulses must undergo simultaneous transverse and longitudinal bunch compression to a coincident focal plane in order to meet the requisite beam power on target [7]. Heavy ion drivers can deliver more intensity to the target per unit length of accelerator by greatly compressing intense ion charge bunches over short distances.

The neutralized transport experiment [8] has demonstrated the feasibility of transverse compression of an intense ion beam by applying a radial focusing angle prior to neutralizing the beam's space-charge and current in a plasma-filled drift region $[9,10]$. When the plasma density $n_{p}$ is much larger than the beam density $n_{b}$ throughout the drift region, quasineutrality is maintained and the beam focuses ballistically to a small spot (few $\mathrm{mm}$ ). When the neutralization level is very high, the focusing is dominated by the transverse emittance, meaning only the inherent transverse temperature of the beam $T_{\perp}$ limits the final beam radius $r_{b}$, due to focusing aberration.
The neutralized drift compression experiment (NDCX) [11] currently studies longitudinal compression in a similar way, by providing plasma to neutralize the beam as it focuses in the axial direction. The primary component upgrade to the previous experiment is a linear induction accelerator [12] with one acceleration gap, which is also known as an induction module. The induction module employs a time-dependent voltage waveform to modify the longitudinal velocity profile of the beam, in order to decrease the initial pulse length and increase the current of the beam. Such focusing can also be longitudinal emittance dominated, due to the inherent longitudinal temperature of the beam $T_{\|}$, provided that the accuracy of the imposed velocity tilt and the level of neutralization by the plasma are both high. The figures of merit for such neutralized drift compression are the compression ratio (maximum final to initial current) and the minimum pulse duration at focus. Compression ratios greater than 50 with fullwidth, half-maximum (FWHM) pulse lengths of $<5 \mathrm{~ns}$ have been measured [13] in the NDCX device, and agree well with reduced numerical models and simulations [14]. Upcoming experiments will attempt to transversely focus a beam with an axial velocity tilt to a submillimeter spot size with coincident focal planes. Particle-in-cell (PIC) simulations [7] and future experiments [15] seek to optimize the amount of current density compression for a given set of experimental constraints. 
The organization of this paper is as follows. The derivation of the equation for the induction module voltage waveform which imposes an ideal velocity tilt on a charge bunch is presented in Sec. II, along with other important parameters. The detailed physics and implications of acceleration gap effects and focusing aberration on optimum longitudinal compression are quantitatively reviewed using PIC simulations in Sec. III, and their dependence on many system parameters is examined. The roles of the finite-size acceleration gap and voltage waveform (Sec. III A), longitudinal beam temperature $T_{\|}$(Sec. IIIB), initial pulse length $t_{p}$ and intended fractional tilt $f$ (Sec. III C), and initial kinetic energy $E_{0}$ and distribution (Sec. III D) are evaluated. Implications pertaining to the simultaneous compression of intense charge bunches are also discussed (Sec. IV). Since imposing the velocity tilt results in the application of a time-dependent radial divergence to the ion beam (Sec. IVA), a strong final-focus solenoid is situated near the end of the drift region in order to transversely focus the beam to a submillimeter spot size coincident with the longitudinal focal plane, and the compression dependence on beam radius $r_{b}(t)$ entering the solenoid is explored (Sec. IV B). A brief summary of the conclusions drawn from the paper is provided in Sec. V.

\section{IDEAL INDUCTION MODULE VOLTAGE WAVEFORM}

There exists an ideal induction module voltage waveform that results in the imposition of a perfect velocity tilt on an ion beam with zero longitudinal temperature across an infinitely thin acceleration gap for achieving maximal longitudinal compression at a desired focal plane [16]. In the classical limit of point particles, there is no upper bound on the longitudinal current compression (or lower bound on the pulse length) under the assumptions mentioned, since an ideal tilt will cause all of the beam particles to arrive at the focal plane at the exact same time. In reality, the current compression cannot be infinite due to longitudinal beam temperature $T_{\|}$, laboratory constraints, and other physical realities that ultimately limit the achievable compression factors and pulse lengths.

In order for all the beam particles to coincidentally arrive at the same longitudinal focal plane, the central and tail portions of the charge bunch are required to travel a greater distance than the head portion in the same amount of time. The time-dependent velocity $v_{b}(t)$ of the particles exiting the gap at time $t$ should be [17]

$$
v_{b}(t)=\frac{v_{h}}{1-\frac{v_{h}}{L_{d}}},
$$

where $v_{h}$ is the decelerated velocity of the head of the beam, $L_{d}$ is the drift length to the focal plane, $t$ is evaluated from $t=0$ to $t=t_{p}$, and $t_{p}$ is the initial pulse length of the beam, which is usually written as $t_{p}=L_{b} / v_{0}$, where $L_{b}$ and $v_{0}$ are the initial bunch length and velocity of the beam before entering the gap. The nonrelativistic energy of the beam is determined from $E_{b}(t)=\frac{1}{2} m_{b} v_{b}^{2}(t)$, where $m_{b}$ is the mass of the beam particles, and the corresponding voltage waveform $V(t)$ for the induction module that produces the desired $E_{b}(t)$ is $V(t)=\frac{1}{q_{b}}\left[E_{b}(t)-E_{0}(t)\right]$, where $q_{b}$ is the charge of the beam particles and $E_{0}(t)$ is the initial energy of the beam ions (usually a constant) entering the gap. For maximum longitudinal compression, the tail of the charge bunch arrives at the focal plane at the same time as the head. The time and drift length required to reach maximal compression are

$$
\begin{aligned}
& t_{h}^{\text {foc }}=\frac{v_{t}}{v_{t}-v_{h}} t_{p} \\
& L_{d}=\frac{v_{h} v_{t}}{v_{t}-v_{h}} t_{p},
\end{aligned}
$$

respectively, where $v_{t}$ is the accelerated velocity of the beam tail relative to $v_{0}$ after it exits the gap at time $t=t_{p}$. Making the assumptions that the $E_{0}$ entering the gap is constant in time, and that equal amounts of head and tail are decelerated and accelerated, respectively, allows the head and tail velocities to be defined as $v_{h} \equiv v_{0}(1-f / 2)$ and $v_{t} \equiv v_{0}(1+f / 2)$, respectively, where the fractional velocity tilt $f$ is defined as

$$
f \equiv \frac{\Delta v_{b}}{v_{0}}=\frac{v_{t}-v_{h}}{v_{0}} .
$$

Then, the time and drift length to longitudinal focus are

$$
\begin{gathered}
t_{h}^{\mathrm{foc}}=\left(\frac{1}{f}+\frac{1}{2}\right) t_{p} \\
L_{d}=v_{0} \frac{\left(1-\frac{f}{2}\right)\left(1+\frac{f}{2}\right)}{f} t_{p}=v_{0}\left(\frac{1}{f}-\frac{f}{4}\right) t_{p} .
\end{gathered}
$$

For the same value of $E_{0}$, larger tilts $f$ give shorter $L_{d}$ than smaller tilts, and longer initial pulse lengths $t_{p}$ give longer $L_{d}$ than shorter pulse lengths.

The time dependence of the velocity as the tilted beam exits the acceleration gap is

$$
v_{b}(t)=v_{0} \frac{\left(1-\frac{f}{2}\right)}{\left[1-\left(\frac{f}{1+\frac{f}{2}}\right)\left(\frac{t}{t_{p}}\right)\right]} .
$$

Then, the required ideal voltage waveform $V(t)$ to timedependently alter the charge bunch energy across the infinitely thin gap is given by [16]

$$
V(t)=\frac{1}{2 q_{b}} m_{b} v_{0}^{2}\left(\left\{\frac{\left(1-\frac{f}{2}\right)}{\left[1-\left(\frac{f}{1+\frac{f}{2}}\right)\left(\frac{t}{t_{p}}\right)\right]}\right\}^{2}-1\right) .
$$

In experiments, the induction module is programmed to attempt the application of as near an ideal waveform as possible. The effects of an imposed nonideal waveform on 
a beam pulse with finite $T_{\|}$across an acceleration gap of finite length have been studied using reduced models [14]. In reality, the induced electric field encountered by the beam has a significant extent in spatial and temporal dimensions, relative to the bunch and pulse lengths of the beam, rather than modifying the beam energy over an infinitely thin slice at one axial location, and gives rise to a number of complications.

\section{ACCELERATION GAP EFFECTS AND LONGITUDINAL FOCUSING ABERRATION}

Kinetic treatments are generally desired in order to realistically compute the experimentally achievable longitudinal compression ratios of ion beams with finite longitudinal temperature $T_{\|}$. Whether the resulting velocity tilt is nearly ideal or not, faster tail particles will overtake slower head particles, and a framework that properly retains the velocity distribution information is required to ascertain the current profiles in the vicinity of the longitudinal focal plane. Knowledge of the complete set of simulated current profiles $I_{b}(z, t)$ of a charge bunch upstream and downstream of the focal plane is valuable when making comparisons to measurements, since diagnostics are situated at one particular axial location, and not necessarily at the longitudinal focal plane.

In general, ion beam trajectories must be evaluated numerically by PIC techniques in order to take into account many of the effects that ultimately determine the quality of the longitudinal focal plane. There is no universally valid analytical model for determining the expected current compression factor and pulse length, or even the current profile $I_{b}(t)$, at the focal plane because of the sensitive dependence on voltage waveform accuracy and finite-size gap geometry, most beam parameters, neutralization processes by the plasma, and other deleterious effects that decrease the current density compression. Simulations of intense beams with nonstationary density and velocity profiles propagating through plasma usually require PIC treatment due to the consideration of complex interactions, such as the nonlinear coupling between the transverse and longitudinal directions, instabilities, collisions between (or ionization of) beam, plasma, and neutral species, and 3D effects. Three-dimensional effects can include such topics as beam off-centering, misalignment of focusing and/or accelerating elements, and beam-beam effects in the interaction regions of colliders or heavy ion fusion drivers. All of the aforementioned issues, and any other nonlinear forces, can give rise to emittance growth of the beam (in either direction) and therefore affect the focusing and compression dynamics.

Sets of PIC simulations are executed in order to quantitatively assess and classify the origin of different sources of longitudinal focal plane degradation involving the acceleration gap [18]. In order to isolate the effects of spacecharge and plasma neutralization processes on the longi- tudinal dynamics from those arising strictly from the gap and initial beam parameters, the perveance and self-fields of the charge bunch are neglected by assuming perfect neutralization of the beam by the plasma in the simulations presented in this paper, which is a valid assumption in the limit $n_{p} \gg n_{b}$ in the drift region (including the focal plane). All of the PIC results presented in this paper are from 2D $\{r, z\}$ LSP simulations [19,20], solve the electromagnetic field equations, treat all particle species as explicit and kinetic, resolve the plasma frequency $\omega_{p}$ and cyclotron frequency $\Omega_{c}$ of all species, are not susceptible to the so-called numerical Debye length instability (because of an energy-conserving push), use a cloud-in-cell model, and conserve energy to good accuracy levels.

\section{A. Dependence on gap size and voltage waveform}

The derivation of the ideal velocity tilt assumed the beam encountered the imposed potential $V(t)$ across an infinitely thin acceleration gap, had no thermal velocity distribution, and was entirely monoenergetic entering the gap. Realistically, an ion beam created in the laboratory must encounter a finite-size gap, always has some finite $T_{\|}$, and might not be entirely monoenergetic entering the gap (or have the appropriate energy for the imparted voltage waveform). Practical engineering considerations such as voltage hold-off and the volt-second capability of the induction module's magnetic flux must be taken into account, which limit the achievable induced electric field. Also, voltage waveform accuracy and its reproduction fidelity are experimental concerns. In order to make better comparison to measurements and provide insight into the physics of neutralized drift compression, a model needs to incorporate the actual spatial and temporal dependence of the electric field induced in the finite-size acceleration gap.

In order to ascertain the effects of the actual acceleration gap in the NDCX on the longitudinal beam dynamics, two types of gap and voltage waveform for modifying the velocity distribution of an $E_{0}=400 \mathrm{keV} K^{+}$ion beam are studied (the maximum $E_{0}$ achievable by the injector in experiments). The first gap is the "infinitely thin" approximation, which is recovered either by injecting the beam from a chosen plane with a prescribed velocity tilt as a time-dependent initial condition, or with an axial electric field strictly imposed over two adjacent grids. The second is the experimental finite-size gap, which begins at the conducting wall radius $r_{w}=3.8 \mathrm{~cm}$, is $3 \mathrm{~cm}$ long in the axial direction, and extends from $z=-22 \mathrm{~cm}$ to $z=$ $-19 \mathrm{~cm}$ in the coordinate system here; the axial coordinate in the laboratory frame extends from $z=-30 \mathrm{~cm}$ to $z=$ $+150 \mathrm{~cm}$, where $z=0$ is the approximate starting location of the neutralizing plasma. In experiments, the plasma drift length can be varied from 1-2.5 m. All simulations involving the $3 \mathrm{~cm}$ gap use an initial beam radius of $r_{b}=$ $2 \mathrm{~cm}$ and convergence angle $\Delta \theta_{r}=-20 \mathrm{mrad}$ at the injection plane $z=-30 \mathrm{~cm}$, the reason for which will be 
discussed shortly. Note that the simulation space begins a few $\mathrm{cm}$ upstream of the acceleration gap with a prescribed beam distribution, rather than modeling the entire accelerator including the ion source and magnetic transport sections.

Two types of voltage waveform are employed for application of the velocity tilt over the two gaps. They are called the "sharp" and "smooth" waveforms and are illustrated in Fig. 1 [note that $V(t)>0$ results in an $-E_{z}(z)$ in this model]. The $V(t)$ shown corresponds to the ideal waveform for an $E_{0}=400 \mathrm{keV} K^{+}$beam with an intended fractional tilt $f=0.5$ over the initial pulse length $t_{p}=300 \mathrm{~ns}$, from $t=200 \mathrm{~ns}$ to $t=500 \mathrm{~ns}$. The drift length to the longitudinal focal plane is predicted by Eq. (6) to be $L_{d}=$ $78.7 \mathrm{~cm}$, or approximately $z=+58.2 \mathrm{~cm}$ in this coordinate system. The sharp waveform ensures that only the ideal electric field is imposed for the duration of the beam's initial pulse length. However, the experimentally realizable waveform requires ramp-up and ramp-down time for its voltage swing, and results in a waveform akin to the smooth one shown in Fig. 1. Therefore, the smooth waveform is the realistically achievable case. Simulations comparing both waveforms shed insight into the contribution to the longitudinal focusing dynamics from the suspected nonideal extra voltage present in the smooth waveform (from $t=0-200 \mathrm{~ns}$ and $t=500-700 \mathrm{~ns}$ in Fig. 1).

A beam with an ideal velocity tilt results from applying the sharp waveform over an infinitely thin gap located at $z=-20.5 \mathrm{~cm}$, which coincides with the center of the $3 \mathrm{~cm}$ gap. The initial cold $K^{+}$beam parameters are $E_{0}=$ $400 \mathrm{keV}, t_{p}=300 \mathrm{~ns}$, and $T_{\|}=0 \mathrm{eV}$ (corresponding to no longitudinal emittance). Since the initial $t_{p}$ is exactly equal to the intended pulse length to be compressed, the entire beam undergoes longitudinal compression. The perfectly velocity-tilted beam with zero longitudinal temperature achieves maximum axial compression at the focal plane $z=+58.25 \mathrm{~cm}$. Since the code evolves point particles, no upper bound on the longitudinal current compression (or lower bound on the pulse length) exists in this case, except for the constraint of the finite time step. A compression ratio of 30000 and minimum pulse length of

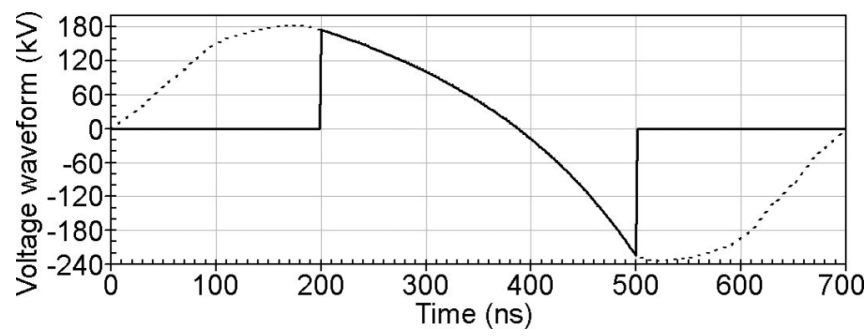

FIG. 1. Representative sharp (solid line) and smooth (dashed line) voltage waveforms used in particle-in-cell simulations. The $V(t)$ shown [refer to Eq. (8)] corresponds to an ideal waveform for an $E_{0}=400 \mathrm{keV} \mathrm{K} K^{+}$beam with fractional tilt $f=0.5$ and initial pulse length $t_{p}=300 \mathrm{~ns}$, from $t=200 \mathrm{~ns}$ to $t=500 \mathrm{~ns}$.
$0.01 \mathrm{~ns}$ are achieved and are the expected classical limiting values, given the $t_{p}=300 \mathrm{~ns}$ and discrete time step $\Delta t=$ $0.01 \mathrm{~ns}$. Since the initial $J_{z}$ is constant, the initial $T_{\|}$is zero, the gap is infinitely thin, and an ideal velocity tilt is perfectly imparted, the axial beam number density profiles vary as $v_{z}^{-1}(z, t)$ and the resulting current profiles in the laboratory frame at fixed axial locations are constant in time (square shaped). Furthermore, the individual current profiles are symmetric about the center of the charge bunch in space and time, and generally about the focal plane. Lastly, the beam focuses at time $t=950 \mathrm{~ns}$, corresponding to $t_{t}^{\text {foc }}=L_{d} / v_{t}=450 \mathrm{~ns}$ after the end of the sharp waveform. No difference in compression is observed using the smooth waveform instead because the extra voltage cannot act across an infinitely thin gap to cause the velocity of any excess beam to lie along the ideal tilt slope.

The simulation just described is repeated with the finitesize gap. In this case, the induced electric field varies as a function of space and time according to the boundary conditions and the sharp waveform, respectively. As revealed in Fig. 2, the cold beam suffers compression degradation compared to the ideal gap case. The compression factor reduces to 2200, and the full-width, half-maximum pulse length increases to $t_{\mathrm{FWHM}}=0.03 \mathrm{~ns}$, as a sole result of the finite-size gap. Also, the beam focuses at the axial location $z=+59.5 \mathrm{~cm}$ at $t=959 \mathrm{~ns}$, approximately $1.25 \mathrm{~cm}$ downstream of the ideal gap focal plane. The additional $9 \mathrm{~ns}$ corresponds to the extra transit time required by the beam particles to travel the extra distance at the average (initial) velocity $v_{0}$. For comparison, the simulation results using an infinitely thin gap are also provided in Fig. 2, with the spatial and temporal offsets included for clarity. More discussion of these results will follow after the description of another simulation.

Next, the sharp waveform is exchanged with the more realistic smooth waveform. Figure 3 presents the difference in the $\left\{z, v_{z}\right\}$ phase space of the beam in both cases involving the finite-size gap, where the initial pulse length

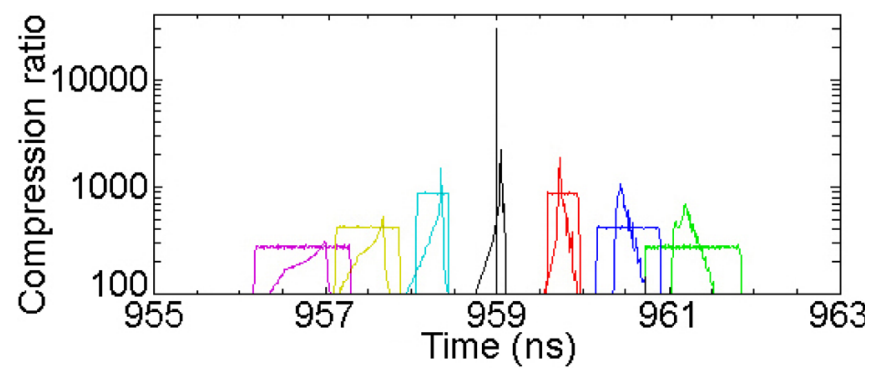

FIG. 2. (Color) Compression ratio $I_{b}(t) / I_{0}$ (log scale) in the laboratory frame at multiple axial locations near the focal plane $(z=+59.5 \mathrm{~cm})$, in steps of $\Delta z=0.1 \mathrm{~cm}$. The beam is initialized with $T_{\|}=0 \mathrm{eV}$ and encounters the sharp waveform across a $3 \mathrm{~cm}$ long gap. The results for the infinitely thin gap case (including the spatial and temporal offsets) are provided for comparison (constant current profiles). 


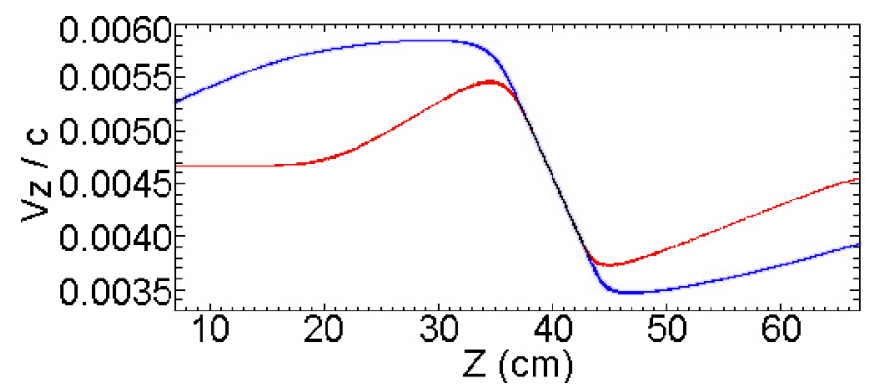

FIG. 3. (Color) $\left\{z, v_{z}\right\}$ phase space at $t=820 \mathrm{~ns}$ for the $T_{\|}=$ $0 \mathrm{eV}$ beam after application of the sharp waveform (red) and smooth waveform (blue) across the finite-size gap.

is much longer than the amount intended for longitudinal compression $\left(t_{p}=300 \mathrm{~ns}\right)$. The axially compressing portion of the beam at this particular time $(t=820 \mathrm{~ns})$ is located in the region of negative slope, from approximately $z=+36 \mathrm{~cm}$ to $z=+44 \mathrm{~cm}$. Decompressing portions of the charge bunch precede and follow the compressing region. Differences in shape are due to the presence of the extra voltage from the finite rise time and fall time of the smooth waveform.

The transit time of the beam particles across the finitesize gap is an important consideration, since it causes the particles to sample a significant temporal range of the voltage waveform. Here, particles moving at velocity $\boldsymbol{v}_{0}$ require approximately $75 \mathrm{~ns}$, which is about $25 \%$ of the intended $t_{p}$ for longitudinal compression, to cross the onaxis $\Delta z \sim 10 \mathrm{~cm}$ affected by the electric field in the gap region due to the boundary conditions. Since the waveform changes appreciably over the transit time, the integrated force experienced by each particle is less than the requisite amount for achieving the desired fractional tilt $f$. Particles within the gap near the beginning ( $t=200 \mathrm{~ns}$, here) and end $(t=500 \mathrm{~ns}$, here) of the waveform do not encounter the appropriate integrated force required to participate in the intended velocity tilt, and contribute to the decompressing head and tail portions of the beam before and after the main compressing pulse, respectively. The spatial extents of the decompressing portions grow significantly later in time (as shown in Fig. 3) compared to the original separation, due to the relative expansion away from the main compressing pulse. It is apparent that the spatial and temporal "shapes" of the electric field in the gap (from the boundary conditions and waveform) impress themselves upon the phase space of the resulting beam.

The result in Fig. 3 demonstrates that the sharp waveform actually compresses less of the beam than the smooth waveform. The extra voltage in the smooth waveform acts on the excess beam to repopulate the low and high velocity space regions in the head and tail, respectively, of the intended tilt (here, the range of the intended $f$ is $v_{z} / c=$ $\{0.0035,0.0058\})$. Therefore, the extra voltage from the ramp-up and ramp-down of the smooth waveform is $a d$ vantageous because of the extra integrated force. The sharp and smooth waveforms, applied across the finite-size gap, result in effective tilts of $f \sim 0.3$ and $f \sim 0.4$, respectively, rather than the intended $f=0.5$ [however, $L_{d}$ does not change according to Eq. (3) or Eq. (6) due to the change in $v_{h}, v_{t}$, and $\left.f\right]$. In other words, the finite-size gap effectively reduces the imparted $f$ from the intended value because of its inability to realize the maximum charge bunch head deceleration and tail acceleration, due to the temporal sampling of more waveform than intended by the particles.

The longitudinal compression results from the sharp and smooth waveform cases for the initial $T_{\|}=0 \mathrm{eV}$ beam across the finite-size gap are shown in Fig. 4. In the linear plot, the difference in compression and pulse length is seen for the current profiles at equally spaced locations near the focal plane. The maximum compression factor at the focal plane increases from 2200 to 2650 due to the larger effective tilt $f$ achieved by the smooth waveform. However, an increased current contribution to the prepulse also occurs due to the extra voltage, as more clearly seen in the log plot of Fig. 4, and the full-width, half-maximum pulse length at the focal plane increases by approximately $17 \%$ to $0.035 \mathrm{~ns}$.

Figure 5 plots the $\left\{z, v_{z}\right\}$ beam phase space, for an initially cold beam, at the focal plane for the case of the sharp waveform across an infinitely thin gap, and compares it to the smooth and sharp waveform cases across the finitesize gap. The cold beam with an ideal tilt from the infinitely thin gap results in an infinite compression (with a vanishing pulse length) in the point particle (and vanishing

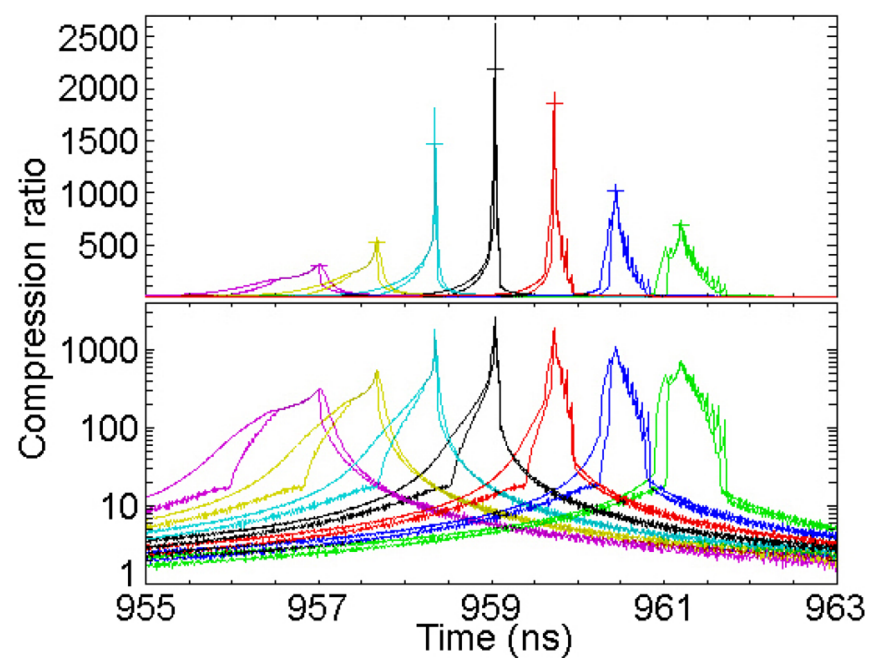

FIG. 4. (Color) Compression ratio $I_{b}(t) / I_{0}$ comparison between smooth (top profiles) and sharp (bottom profiles) waveforms on linear (top) and $\log$ (bottom) scales in the laboratory frame at multiple axial locations near the focal plane $(z=+59.5 \mathrm{~cm})$, in steps of $\Delta z=0.1 \mathrm{~cm}$. The initial $T_{\|}=0 \mathrm{eV}$ beams are tilted across the finite-size gap. Nonphysical horizontal bars are added on the linear scale plot to demarcate the excess compression in the smooth waveform case. The log scale plot shows the increased amount of current in the prepulse of the smooth waveform case. 


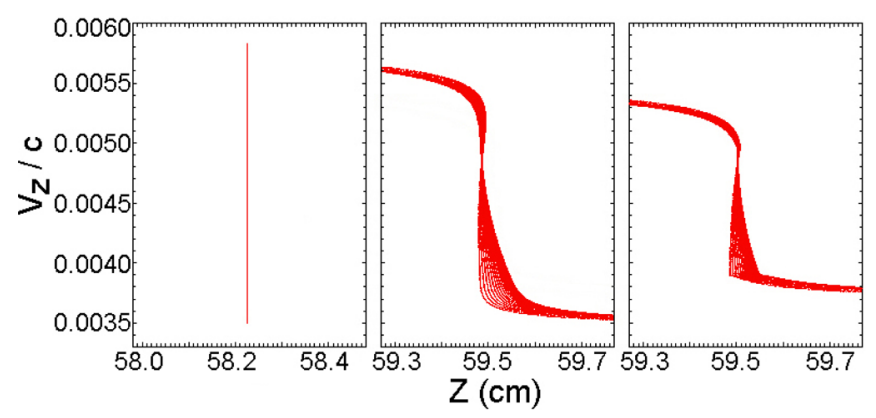

FIG. 5. (Color) $\left\{z, v_{z}\right\}$ phase space at the focal plane for the $T_{\|}=0 \mathrm{eV}$ beam using the sharp waveform across an infinitely thin gap (left), and smooth (center) and sharp (right) waveforms across the finite-size gap. Longitudinal focus occurs at $z=$ $+58.25 \mathrm{~cm}$ and $t=950 \mathrm{~ns}$ for the former case, and at $z=$ $+59.5 \mathrm{~cm}$ and $t=959 \mathrm{~ns}$ for the latter cases.

time step) limit, as discussed earlier and shown on the left of Fig. 5, where all of the beam particles arrive at the focal plane $(z=+58.25 \mathrm{~cm})$ at the same time $(t=950 \mathrm{~ns})$. The center and right frames of Fig. 5 illustrate the effects of a finite-size gap and the use of smooth and sharp waveforms, respectively, on an initially cold beam.

Two important conclusions are drawn from the center and right plots of Fig. 5. First, the imposition of $V(t)$ across a finite-size gap imparts velocity spread akin to an effective longitudinal temperature on an otherwise initially cold beam with no space-charge. Therefore, a finite upper (lower) bound on the maximum compression (minimum pulse length) is set, due to longitudinal focusing aberration. The origin of this $2 \mathrm{D}$ effect is found in the coupling of the transverse and longitudinal dynamics of the beam as it traverses the finite-size gap. Radial components of the electric field exist wherever $\partial E_{z}(r, z) / \partial z \neq 0$, as illustrated in Fig. 6. The time-dependent nature of $V(t)$ and the transit time across the gap imply an imbalance of integrated $E_{r}(r, z, t)$ felt by each particle. In other words, the radial fields modify the $v_{r}$ distribution of the beam nonuniformly. As a result, particles sample unequal amounts of integrated $E_{z}(r, z, t)$ across the gap, instead of the same radially independent integrated amount. During

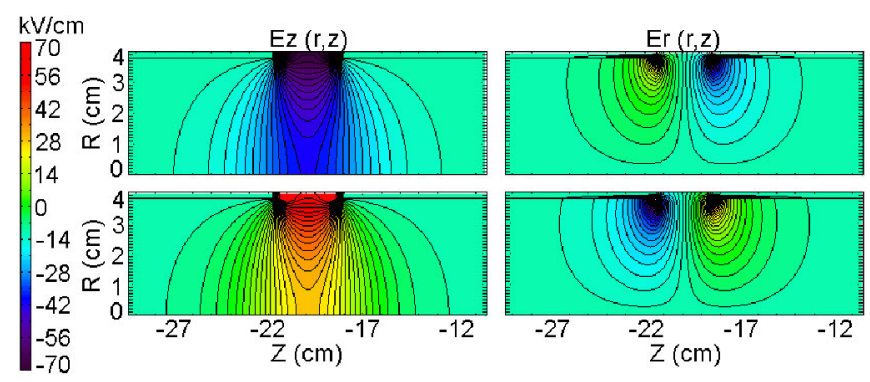

FIG. 6. (Color) $\{r, z\}$ isocontour plots of $E_{z}(r, z)$ at $t=200 \mathrm{~ns}$ (top left) and $t=500 \mathrm{~ns}$ (bottom left) and $E_{r}(r, z)$ at $t=200 \mathrm{~ns}$ (top right) and $t=500 \mathrm{~ns}$ (bottom right) near the $3 \mathrm{~cm}$ acceleration gap. All plots are on the same linear scale. the compressing part of the voltage waveform $[d V(t) / d t<$ $0]$, beam particles at larger radii encounter a stronger imbalance of $E_{r}$ fields, acquire more time-dependent changes to $v_{r}$, and are subsequently decelerated [for times when $V(t)>0$ and $E_{z}(t)<0$ ] or accelerated [for times when $V(t)<0$ and $E_{z}(t)>0$ ] in the axial direction more than particles initially closer to the $r=0$ axis. When the voltage waveform polarity switch occurs, and particles farther off-axis switch from more axial deceleration to acceleration, it causes the $\mathrm{X}$-shaped spread in the $v_{z}$ distribution, as apparent in the center and right plots of Fig. 5. Therefore, due to the transverse movement of the particle trajectories within the gap, the beam acquires a longitudinal velocity spread similar to temperature $T_{\|}$.

The effective $T_{\|}$increase acquired by the beam is dependent upon the gap geometry, voltage waveform, and beam parameters, such as axial velocity, radius, convergence angle, and longitudinal emittance, and will be quantitatively determined later. Note that the reduced velocity tilt $f$ suffered by a beam crossing a finite-size gap should also be interpreted as an increase in effective longitudinal temperature, since the drift length $L_{d}$ to the focal plane does not change with the reduced $f$ value according to Eq. (6). As will be shown later, the presence of additional $T_{\|}$decreases the compression ratio and increases the pulse length at focus without changing the axial location of the focal plane.

The effective increase in $T_{\|}$is also dependent upon the transverse beam convergence entering the gap (quoted as $\Delta \theta_{r}$ in radians as the beam radius $r_{b}$ over the transverse focusing length $L_{r}$ ), in addition to particle radius. Beams undergoing a transverse final focus generally have radial convergent angles much larger than the transverse thermal spread, as they exit the transport section and enter the gap, for transverse confinement as well as focusing (otherwise no focusing would be achieved). The $E_{r}$ imbalance across the gap provides an average time-dependent radial defocusing force to all portions of the beam during the compressing $[d V(t) / d t<0]$ part of the waveform [18,21]. Radial motion causes the sampling of unequal amounts of $E_{z}(r, z, t)$ across the gap; therefore, a beam entering the gap with a trajectory that is radially converging will have some of its initial convergence offset by the timedependent radial defocusing force. Then, the beam acquires less effective $T_{\|}$(and longitudinally compresses better) because it samples less integrated $E_{z}(r, z, t)$ imbalance, compared to the case without an applied convergence.

The effect is shown in Fig. 7 for the beam parameters under previous consideration, with $r_{b}=2 \mathrm{~cm}$ and a convergence angle of $\Delta \theta_{r}=-20 \mathrm{mrad}$. The beam's $v_{r}$ distribution varies radially as $-v_{r}(r)=-v_{z}^{0}\left(r / r_{b}\right)\left(r_{b} / L_{r}\right)$ for $r=0$ to $r=r_{b}$. The case without a convergence angle suffered more gap-induced effective $T_{\|}$increase due to increased radial movement within the gap compared to 


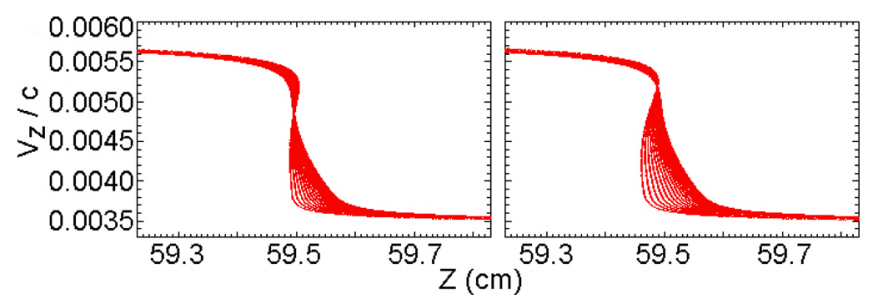

FIG. 7. (Color) $\left\{z, v_{z}\right\}$ phase space at the focal plane for the $T_{\|}=0 \mathrm{eV}, r_{b}=2 \mathrm{~cm}$ beam with a $-20 \mathrm{mr}$ convergence angle at $z=-30 \mathrm{~cm}$ (left) and no convergence angle (right) using the smooth waveform across the finite-size gap. The compression ratios and full-width, half-maximum pulse lengths at focus are 2650 and 0.035 ns compared to 1500 and 0.065 ns.

the case with an -20 mrad angle at the plane of injection $z=-30 \mathrm{~cm}$. The $r_{b}=2 \mathrm{~cm}, \Delta \theta_{r}=-20 \mathrm{mrad}$ case is approximately the optimum radial initial condition for the average offsetting of the time-dependent defocusing force for the considered waveforms, and is used in all of the simulations involving the finite-size gap.

The second conclusion drawn from Fig. 5 is that the reduction of the intended tilt $f$, by the smooth and sharp waveforms acting across the finite-size gap, affects the faster tail particle velocities more than those in the slower head. From the left frame of Fig. 5, the expected minimum head and maximum tail velocities are $v_{z}^{h} / c=0.00350$ and $v_{z}^{t} / c=0.00583$, respectively. From the center and right frames of Fig. 5, the realized maximum tail velocities are reduced compared to the infinitely thin gap case, more so than the difference between the minimum head velocities compared to the same. This effect occurs because accelerated tail particles have reduced transit times relative to the decelerated head particles, and therefore the integrated energy gain by the tail particles is not equal and opposite to the integrated energy loss by the head particles, since the energy modulation from the waveform is not imparted instantaneously.

In reality, not only does the acceleration gap have finite size, but a charge bunch has nonzero initial longitudinal temperature. Therefore, compression factors and pulse lengths at focus will be further reduced and increased, respectively, due to larger amounts of axial focusing aberration relative to the cold beam cases. In order to quantify how finite $T_{\|}$further reduces the established upper limits on longitudinal compression of a cold beam with NDCXrelevant parameters, additional simulations including the nonzero initial longitudinal emittance of an ion beam are carried out.

\section{B. Dependence on longitudinal temperature $\boldsymbol{T}_{\|}$}

The NDCX device is equipped with an injector capable of generating a high-brightness, low-emittance, and variable-perveance $K^{+}$ion beam with $E_{0} \leq 400 \mathrm{keV}$ of directed energy [8]. A beam's brightness is proportional to the ratio of its current to the square of its transverse emittance, where the beam's transverse emittance is a measure of its area in phase space, and is related to its internal transverse temperature according to [22]

$$
\epsilon_{n}^{4 \mathrm{rms}}=2 r_{b} \sqrt{\frac{T_{\perp}}{m_{i} c^{2}}},
$$

for a nonrelativistic, uniform round beam with a Maxwellian velocity distribution. Here, $r_{b}$ is the beam radius, $T_{\perp}$ is the transverse temperature, and $m_{i} c^{2}$ is the rest energy of the beam. The normalized four-times-rms transverse effective emittance is given by Eq. (9) in units of $\mathrm{m}$-rad (typically quoted in cm-mrad or mm-mrad). The longitudinal emittance is similarly defined with the longitudinal temperature $T_{\|}$and axial rms bunch half-length $z_{b}$ instead. Liouville's theorem ensures that the normalized emittance is conserved in the absence of nonlinear forces and coupling forces between different coordinate directions; in practice, Eq. (9) is a theoretical lower limit because nonlinear dynamics, collisions, instabilities, and other effects can cause beam emittance growth in experiments [22]. Beams with low emittances are desirable because they experience less aberration of the focal plane.

In another simulation, the sharp waveform is employed over an infinitely thin gap and causes a fractional tilt of $f=0.5$ on a beam whose initial parameters are $E_{0}=$ $400 \mathrm{keV}$, and $t_{p}=300 \mathrm{~ns}$, with a $T_{\|}=T_{\perp}=0.2 \mathrm{eV}$ Maxwellian distribution just upstream of the acceleration gap. The chosen beam temperatures are consistent with measurements in the NDCX device. The current compression results near the focal plane are given in Fig. 8, alongside the cold beam results from Fig. 2 involving the same gap and waveform. The maximum compression ratio for the $T_{\|}=0.2 \mathrm{eV}$ case is 400 with a full-width, halfmaximum pulse length of $t_{\mathrm{FWHM}} \sim 0.60 \mathrm{~ns}$.

The results in Fig. 8 show that the compression in the $T_{\|}=0.2 \mathrm{eV}$ case is significantly affected by aberration, which is manifest as a smearing of the longitudinal focal

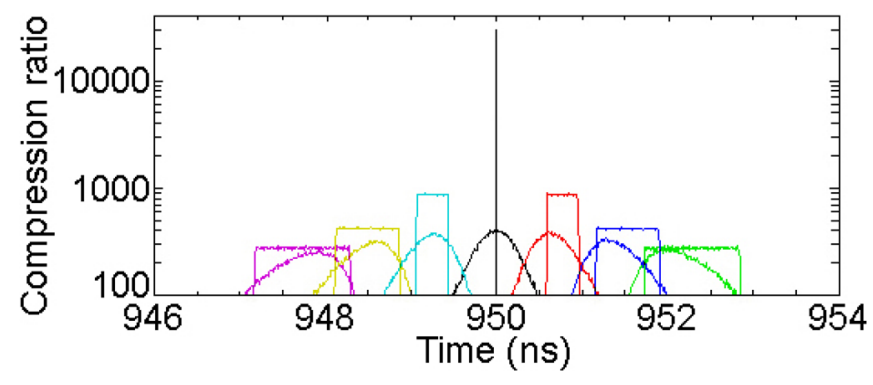

FIG. 8. (Color) Compression ratio $I_{b}(t) / I_{0}$ (log scale) comparison in the laboratory frame at multiple axial locations near the focal plane $(z=+58.25 \mathrm{~cm})$, in steps of $\Delta z=0.1 \mathrm{~cm}$, for beams initialized with $T_{\|}=0 \mathrm{eV}$ (constant current profiles) and $T_{\|}=0.2 \mathrm{eV}$ (varying current profiles), both of which are ideally tilted using the sharp waveform across an infinitely thin gap. 
plane because of the axial velocity spread. Focusing aberration increases the level of tolerance (at the cost of compression) for aligning a diagnostic or target with the focal plane: more $T_{\|}$causes the peak current to vary less sensitively as a function of axial coordinate. The presence of longitudinal temperature alone (even under this idealized case) results in asymmetric individual $I_{b}(t) / I_{0}$ profiles in time about their respective peak values away from the focal plane. Slower head particles suffer increased levels of focusing aberration (discussed below) and are sampled for longer durations at one particular axial location in the laboratory frame. They contribute more current to the prepulse for times before peak compression, whereas the opposite is true for the faster tail particles contributing to the postpulse. After the focal plane has passed, the situation reverses and the prepulse is reduced relative to the postpulse because the head particles are surpassed by the tail particles. Since the exact amount of initial beam pulse is perfectly tilted in this case, the profiles and the envelope of the peak current (from one profile to the next) are symmetric about the focal plane itself.

Returning to Figs. 2 and 4, the profiles in those plots are asymmetric due to the effective nonuniform $T_{\|}$imparted to an initially cold beam by the finite-size gap (refer also to Fig. 5). Likewise, the individual current profiles and the envelope of the peak current are not generally symmetric about the focal plane in those figures because more than the prescribed initial pulse length $t_{p}=300 \mathrm{~ns}$ (according to the waveform) of beam is used. Downstream of the focal plane, the tail of the beam passes the head, causing the formation of different current profile features due to the multiple-valued nature of the $\left\{z, v_{z}\right\}$ beam phase space at fixed $z$ (similar to a $Z$-shape, if one considers the afterfocus analogs of Figs. 3 and 5).

The $\left\{z, v_{z}\right\}$ phase space 2 ns upstream of the focal plane, at the focal plane, and $2 \mathrm{~ns}$ downstream of the focal plane for the $T_{\|}=0.2 \mathrm{eV}$ case using the sharp waveform across an infinitely thin gap are presented in Fig. 9. The distance traveled in $2 \mathrm{~ns}$ by the beam is approximately $0.2 \mathrm{~cm}$; therefore, the yellow, black, and dark blue current profiles of the $T_{\|}=0.2 \mathrm{eV}$ case in Fig. 8 are directly related to the

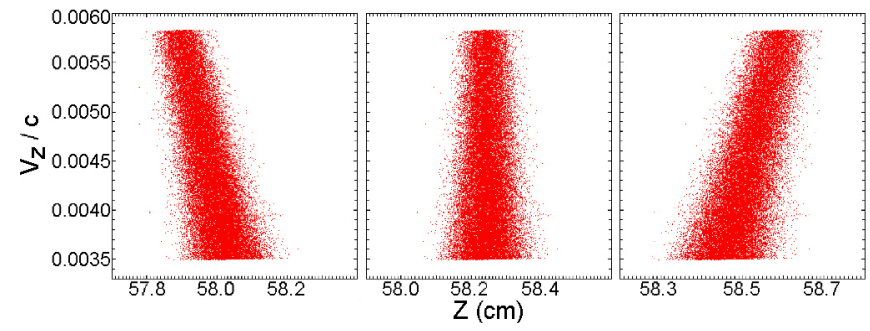

FIG. 9. (Color) $\left\{z, v_{z}\right\}$ phase space for the $T_{\|}=0.2 \mathrm{eV}$ beam, using the sharp waveform across an infinitely thin gap, 2 ns before arrival at the focal plane (left), at the focal plane (center), and $2 \mathrm{~ns}$ after passing the focal plane (right). Focus occurs at $z=+58.25 \mathrm{~cm}$ and $t=950 \mathrm{~ns}$. left, center, and right frames of Fig. 9, respectively. Inspection of the two figures highlights the contribution of the velocity distribution (Maxwellian, here) from an ideally tilted beam to the shape of the current profile at a fixed location. Additional simulations regarding the role of the type of thermal distribution on the current compression will be discussed later.

A significant effect apparent in Fig. 9 is that slower head particles suffer more aberration due to finite- $T_{\|}$effects during the longitudinal focusing process, a result of the fact that the thermal velocity is always a greater fraction of head velocities compared to those in the tail. Taking this effect into account, the contribution of aberration for an ion beam with finite initial $T_{\|}$(which has been ideally velocity tilted) to the temporal smearing of the longitudinal focal plane can be calculated as follows.

Consider an ion beam with a Waterbag axial velocity distribution and thermal velocity $v_{\text {th }} \ll v_{0}$, where the socalled Waterbag distribution is one with uniform-density and equally populated between $v_{0}-v_{\text {th }}$ and $v_{0}+v_{\text {th }}$. The effective drift length to the focal plane can be calculated from Eq. (3) to be

$$
L_{d}^{h+}=\frac{\left(v_{h}+v_{\mathrm{th}}\right) v_{t}}{v_{t}-\left(v_{h}+v_{\mathrm{th}}\right)} t_{p}
$$

for the head particles moving with the slightly faster velocity $v_{h}+v_{\text {th }}$ and

$$
L_{d}^{h-}=\frac{\left(v_{h}-v_{\mathrm{th}}\right) v_{t}}{v_{t}-\left(v_{h}-v_{\mathrm{th}}\right)} t_{p}
$$

for the head particles moving with the slightly slower velocity $v_{h}-v_{\text {th }}$. Then, the focal plane is no longer infinitely thin at one axial location $L_{d}$ downstream of the gap, but is smeared about that location due to the beam's longitudinal temperature. The width of the range about $L_{d}$ for the head of the charge bunch is

$$
w^{h} \equiv L_{d}^{h+}-L_{d}^{h-}=2 \frac{v_{t}^{2}}{\left(v_{t}-v_{h}\right)^{2}-v_{\mathrm{th}}^{2}} v_{\mathrm{th}} t_{p} .
$$

Similarly, the intended focal plane takes on a range of values whose width about $L_{d}$ for the tail of the charge bunch is

$$
w^{t}=2 \frac{v_{h}^{2}}{\left(v_{t}-v_{h}\right)^{2}-v_{\mathrm{th}}^{2}} v_{\mathrm{th}} t_{p} .
$$

The excess focusing aberration suffered by the slower head particles compared to the faster tail particles is then

$$
w^{h}-w^{t}=2 \frac{v_{t}^{2}-v_{h}^{2}}{\left(v_{t}-v_{h}\right)^{2}-v_{\mathrm{th}}^{2}} v_{\mathrm{th}} t_{p},
$$

and can be rewritten in terms of the initial beam velocity $v_{0}$ and the fractional tilt $f$, using the definitions of $v_{h}$ and $v_{t}$ and their associated assumptions, as 


$$
w^{h}-w^{t}=\frac{4}{f} \frac{1}{1-\left(\frac{v_{\mathrm{th}}}{f v_{0}}\right)^{2}} v_{\mathrm{th}} t_{p}
$$

The effective temperature of a Waterbag distribution is defined as

$$
T_{W} \equiv m_{b} \int_{-v_{\text {th }}}^{+v_{\text {th }}} \frac{d \mathbf{v}}{2 v_{\text {th }}} \mathbf{v}^{2}=\frac{1}{3} m_{b} v_{\mathrm{th}}^{2}
$$

whereas the temperature of a Maxwellian distribution is defined as

$$
T_{M} \equiv m_{b} \int_{-\infty}^{+\infty} \frac{d \mathbf{v}}{\sqrt{2 \pi} v_{\text {th }}} \mathbf{v}^{2} \exp \left(-\frac{\mathbf{v}^{2}}{2 v_{\mathrm{th}}^{2}}\right)=m_{b} v_{\mathrm{th}}^{2}
$$

and $T_{M}=3 T_{W}$ for the same value of $v_{\text {th }}$. To analyze PIC simulations involving beams with Maxwellian distributions, the factor of $\sqrt{3}$ in $v_{\mathrm{th}}\left(v_{\mathrm{th}}^{M}=v_{\mathrm{th}}^{W} / \sqrt{3}\right)$ should be taken into account in Eqs. (10)-(15).

For the parameters of the simulations presented in Figs. 8 and 9, the widths $w^{h}$ and $w^{t}$ about $L_{d}$ are 0.151 and $0.054 \mathrm{~cm}$, respectively, and agree well with the center frame of Fig. 9, which quantitatively shows the same 2.8 ratio of widths $\left(w^{h} / w^{t}\right)$ between the slowest and fastest particles. The full-width, half-maximum pulse length of the ideally tilted beam with finite initial longitudinal temperature at the focal plane is approximately equal to the weighted average

$$
t_{\mathrm{FWHM}}=\frac{1}{2}\left[\frac{\left(\frac{w^{h}}{v_{h}}\right) w^{h}+\left(\frac{w^{t}}{v^{t}}\right) w^{t}}{w^{h}+w^{t}}\right],
$$

which is $t_{\mathrm{FWHM}}=0.58 \mathrm{~ns}$ for the parameters here, in close agreement with the full-width, half-maximum pulse length of $t_{\mathrm{FWHM}} \sim 0.60 \mathrm{~ns}$ plotted in the black current profile in Fig. 8 for the $T_{\|}=0.2 \mathrm{eV}$ case.

Two additional simulations are executed in order to compare the compression results from the sharp and smooth waveforms imposed over the finite-size gap to the simulation involving the sharp waveform over an infinitely thin gap. The current profiles near the focal plane using the two waveforms applied to the $T_{\|}=0.2 \mathrm{eV}$ beam over the finite-size gap are shown in Fig. 10 on a linear scale and are accompanied by the infinitely thin gap results of the same $T_{\|}=0.2 \mathrm{eV}$ beam (previously plotted on a $\log$ scale in Fig. 8). The aforementioned spatial and temporal offsets between the two gaps are included for clarity. As before, the smooth waveform effectively compresses more beam than the sharp waveform acting over the finite-size gap. The maximum compression ratio and full-width, halfmaximum pulse length for the infinitely thin gap case are 400 and $t_{\mathrm{FWHM}} \sim 0.6 \mathrm{~ns}$, respectively. The corresponding sharp and smooth waveform results over the finite-size gap are approximately 235 compared to 320 , and $0.65 \mathrm{~ns}$ compared to $0.70 \mathrm{~ns}$, respectively. The differences in the finite-

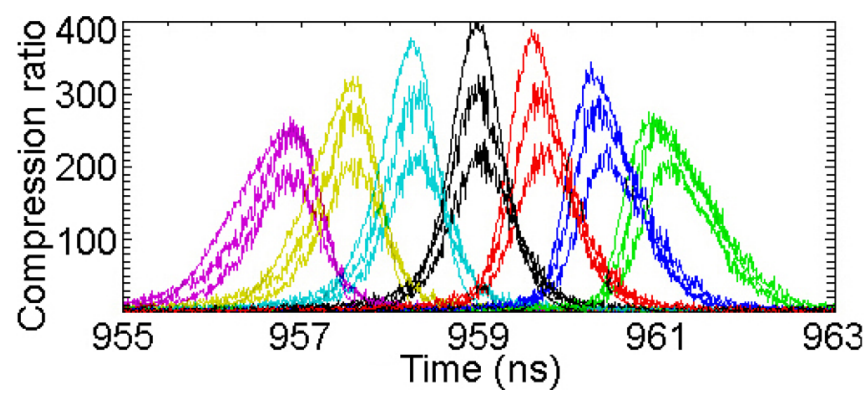

FIG. 10. (Color) Compression ratio $I_{b}(t) / I_{0}$ comparison between smooth (middle profiles) and sharp (bottom profiles) waveforms in the laboratory frame at multiple axial locations near the focal plane $(z=+59.5 \mathrm{~cm})$, in steps of $\Delta z=0.1 \mathrm{~cm}$. The beams initialized with $T_{\|}=0.2 \mathrm{eV}$ are velocity tilted across the $3 \mathrm{~cm}$ long gap. The infinitely thin gap case results (including the spatial and temporal offsets) from Fig. 8 are provided for comparison (top profiles).

size gap cases relative to the infinitely thin gap case are due to the decreased effective tilts $f$ (acting as effective $T_{\|}$ increases), compared to the intended $f=0.5$, and associated increased levels of focusing aberration.

The $\left\{z, v_{z}\right\}$ beam phase space for the three cases described above at the longitudinal focal plane are provided in Fig. 11, and should be compared to the complementary $T_{\|}=0 \mathrm{eV}$ case results in Fig. 5. The two main effects of the finite-size gap are evident again in these simulations with an increased axial velocity spread. The slower head particles experience more longitudinal focusing aberration relative to the faster tail particles, and the decrease in maximum tail velocities from the ideal case is relatively larger than the corresponding increase in minimum head velocities from the ideal case. The tilt is again reduced from the intended $f=0.5$ to $f \sim 0.4$ and $f \sim 0.3$ for the smooth and sharp waveform cases, respectively, across the finite-size gap. Because of the initial $T_{\|}$increase, the compression factors for the sharp and smooth waveforms across the finite-size gap precipitously drop from 2200 to

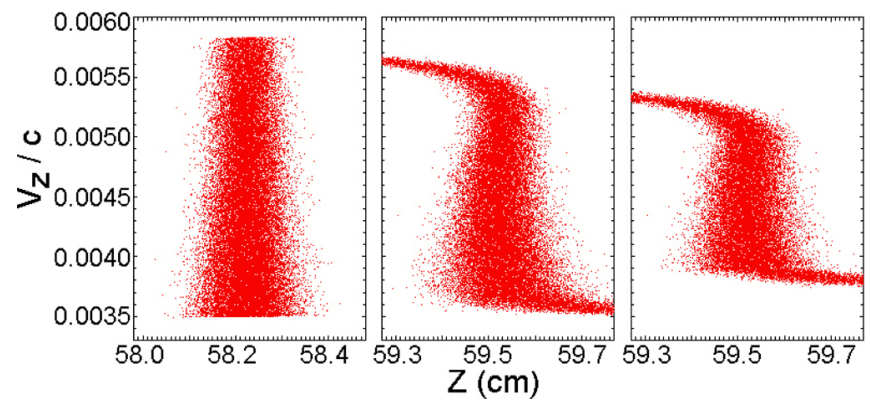

FIG. 11. (Color) $\left\{z, v_{z}\right\}$ phase space at the focal plane for the $T_{\|}=0.2 \mathrm{eV}$ beam using the sharp waveform across an infinitely thin gap (left), and smooth (center) and sharp (right) waveforms across the finite-size gap. Focus occurs at $z=+58.25 \mathrm{~cm}$ and $t=950 \mathrm{~ns}$ for the former case, and at $z=+59.5 \mathrm{~cm}$ and $t=$ $959 \mathrm{~ns}$ for the latter cases. 
TABLE I. Compression dependence on initial $T_{\|}$for $E_{0}=$ $400 \mathrm{keV}, t_{p}=300 \mathrm{~ns}$, and $f=0.5$ using the smooth waveform across the $3 \mathrm{~cm}$ long gap.

\begin{tabular}{lrccc}
\hline \hline$T_{\|}$ & $I_{b}^{\max } / I_{0}$ & $t_{\mathrm{FWHM}}$ & $t_{\mathrm{FWHM}}$ [Eq. (18)] & $T_{\|}^{\text {inf }}$ [Eq. (18)] \\
\hline $0 \mathrm{eV}$ & 2650 & $0.035 \mathrm{~ns}$ & $0 \mathrm{~ns}$ & $7.5 \times 10^{-4} \mathrm{eV}$ \\
$0.1 \mathrm{eV}$ & 430 & $0.51 \mathrm{~ns}$ & $0.41 \mathrm{~ns}$ & $0.16 \mathrm{eV}$ \\
$0.2 \mathrm{eV}$ & 320 & $0.70 \mathrm{~ns}$ & $0.58 \mathrm{~ns}$ & $0.30 \mathrm{eV}$ \\
$0.4 \mathrm{eV}$ & 230 & $1.01 \mathrm{~ns}$ & $0.81 \mathrm{~ns}$ & $0.62 \mathrm{eV}$ \\
$0.8 \mathrm{eV}$ & 160 & $1.40 \mathrm{~ns}$ & $1.15 \mathrm{~ns}$ & $1.19 \mathrm{eV}$ \\
$1.6 \mathrm{eV}$ & 115 & $2.05 \mathrm{~ns}$ & $1.63 \mathrm{~ns}$ & $2.55 \mathrm{eV}$ \\
\hline \hline
\end{tabular}

235 and 2650 to 320 , respectively, and the full-width, halfmaximum pulse lengths increase from 0.03 to $0.65 \mathrm{~ns}$ and 0.035 to $0.70 \mathrm{~ns}$, respectively. The reduction in $f$ causes the apparent increase in effective $T_{\|}$.

Simulations involving a range of initialized Maxwellian longitudinal temperatures, for the case of the smooth voltage waveform across the finite-size gap, investigate the dependence of the current ratio on the initial longitudinal emittance of the charge bunch. The initialized longitudinal temperatures are $T_{\|}=0.1 \mathrm{eV}, 0.2 \mathrm{eV}, 0.4 \mathrm{eV}, 0.8 \mathrm{eV}$, and $1.6 \mathrm{eV}$. The compression ratios achieved at the focal plane and the full-width, half-maximum pulse lengths are presented in Table I. The data in Table I correspond to the peak current profiles for those cases plotted together in Fig. 12 for comparison. The $T_{\|}=0 \mathrm{eV}$ case is included in Table I and Fig. 12 for reference.

From Table I, the compression factor decreases and the minimum pulse length increases with an approximate square root dependence on the initial longitudinal temperature ( $T_{\|}^{-0.48}$ and $T_{\|}^{0.5}$, respectively) as expected, since $t_{\mathrm{FWHM}} \sim v_{\text {th }} \sim \sqrt{T_{\|}}$according to Eq. (18) for $v_{\text {th }} \ll v_{h}$, $v_{t}$. The minimum full-width, half-maximum pulse length of a beam, which acquires an ideal velocity tilt across an infinitely thin gap and is perfectly neutralized, will be limited by the initial longitudinal temperature (emittance) according to Eq. (18). The predicted $t_{\mathrm{FWHM}}$ from that equation is shown in Table I. Although Eq. (18) includes

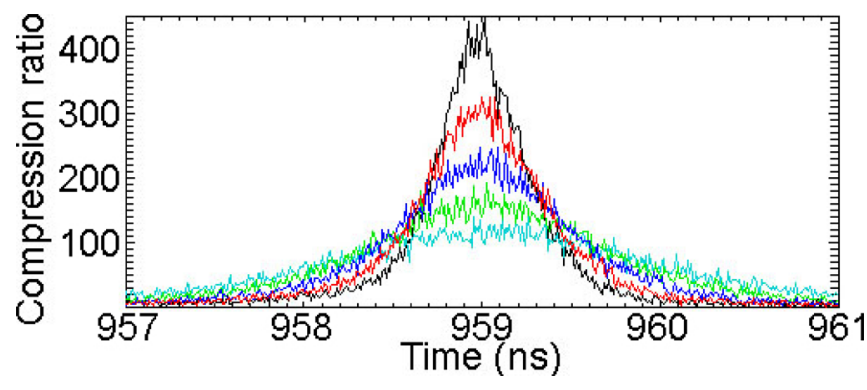

FIG. 12. (Color) Compression ratio $I_{b}(t) / I_{0}$ comparison using the smooth waveform across the finite-size gap in the laboratory frame at the focal plane $(z=+59.5 \mathrm{~cm})$ for initialized $T_{\|}=$ $0.1 \mathrm{eV}$ (black), $0.2 \mathrm{eV}$ (red), $0.4 \mathrm{eV}$ (dark blue), $0.8 \mathrm{eV}$ (green), and $1.6 \mathrm{eV}$ (light blue). the linearly weighted average effect of the longitudinal aberration experienced by all the beam particles, the equation does not include the finite-size gap or smooth waveform effects (both of which increase the beam's effective $T_{\|}$), as shown by the discrepancy between the third and fourth columns of Table I. Note that the difference between those columns also increases approximately with the square root of the initial $T_{\|}$.

The inferred initial beam temperatures $T_{\|}^{\text {inf }}$ required to produce the value of $t_{\mathrm{FWHM}}$ from the PIC simulations using Eq. (18) are presented in the fifth column of Table I. The inferred longitudinal temperatures are $55 \% \pm 5 \%$ higher on average than the initial $T_{\|}$used in simulations involving the $3 \mathrm{~cm}$ gap. The elevated values indicate the required presence of an additional constant $\left(\sqrt{1.55}\right.$, here) in the $v_{\text {th }}$ variable of Eqs. (12) and (13), in order to take into account the combined effects of the gap size and smooth waveform, for the set of parameters considered here. Recall that the infinitely thin gap case results in $t_{\mathrm{FWHM}}=0.6 \mathrm{~ns}$, and the finite-size gap with a smooth waveform case results in $t_{\text {FWHM }}=0.7 \mathrm{~ns}$ for $T_{\|}=0.2 \mathrm{eV}$, whereas Eq. (18) predicts $t_{\mathrm{FWHM}}=0.58 \mathrm{~ns}$. Therefore, $17 \%$ of the $T_{\|}$discrepancy is due to error in the equation (the averaging procedure linearly weights the velocities from $v_{h}$ to $v_{t}$, but the tilt deviates from linearity later in the pulse in this case) and $83 \%$ of the discrepancy is due to the increased effective $T_{\|}$from the gap and waveform. Additional simulations involving other acceleration gap sizes only support a weak dependence on the length of the gap. However, the gap size and transit time of the particles should be considerably smaller than the initial bunch length $L_{b}$ and pulse length $t_{p}$, respectively, for effectively imparting an intended fractional tilt $f$.

The amount of effective $T_{\|}$imparted to an $E_{0}=$ $400 \mathrm{keV}$ beam with nonzero initial $T_{\|}$as it encounters the smooth waveform for $t_{p}=300 \mathrm{~ns}$ and $f=0.5$ across a finite-size gap can be determined by comparing to cases involving an infinitely thin gap. As shown in Fig. 13, four cases of initial $T_{\|}$compare the case of the finite-size gap employing the smooth waveform to the infinitely thin gap case. Simulations initialized with $T_{\|}=0.2 \mathrm{eV}, 0.4 \mathrm{eV}$, $0.8 \mathrm{eV}$, and $1.6 \mathrm{eV}$ each result in an approximate $20 \%$ relative decrease in maximum compression ratio and increase in full-width, half-maximum pulse length for the finite-size gap and smooth waveform case compared to the infinitely thin gap case, as plotted in Fig. 13. Therefore, the effective longitudinal temperature difference between the two cases, due to the reduced $f$ from finite-size gap and smooth waveform effects, is approximately $45 \%$ and in very good agreement with the previous results, which state that the compression factor decreases with the square root of increasing $T_{\|}$. As in the previous paragraph, the inferred $T_{\|}^{\mathrm{inf}}$ are $\sim 55 \%$ higher on average in Table I, and $\sim 83 \%$ of that discrepancy $(\sim 45 \%)$ is due to the effects of the finite- 


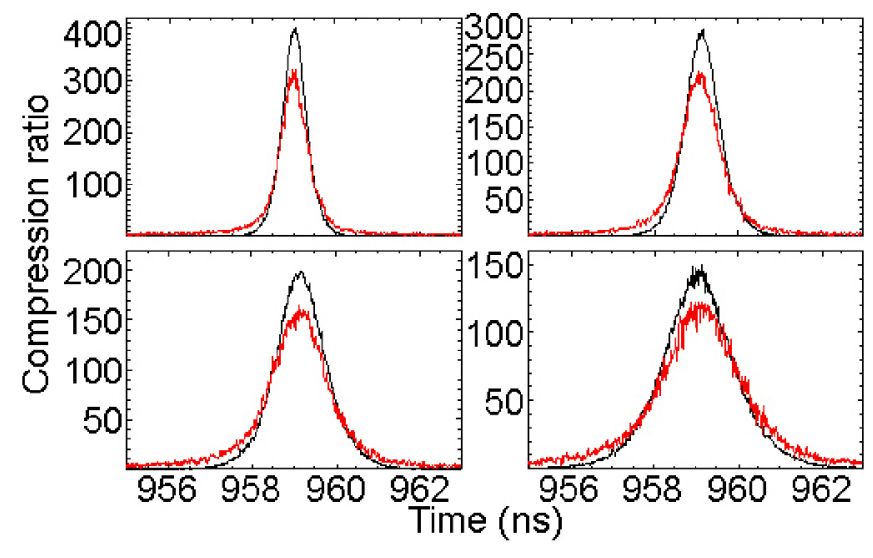

FIG. 13. (Color) Compression ratio $I_{b}(t) / I_{0}$ comparison between the sharp waveform across the infinitely thin gap (black) and smooth waveform across the finite-size gap (red) in the laboratory frame at the focal plane (including the temporal and spatial offsets) for initialized $T_{\|}=0.2 \mathrm{eV}$ (top left), $0.4 \mathrm{eV}$ (top right), $0.8 \mathrm{eV}$ (bottom left), and $1.6 \mathrm{eV}$ (bottom right).

size gap and smooth waveform. As a check, simulations comparing $T_{\|}=0.1 \mathrm{eV}$ in the finite-size gap case to $T_{\|}=$ $0.145 \mathrm{eV}$ in the infinitely thin gap case confirm the same compression result. In summary, for the parameters considered here, the $3 \mathrm{~cm}$ gap and smooth waveform together add the equivalent of approximately $45 \%$ of the initial $T_{\|}$to the resulting effective longitudinal temperature by reducing the achieved $f$ relative to the intended $f$, and coupling the transverse and longitudinal phase space as the beam traverses the gap.

Analytical neutralized drift compression equations exist [23] for calculating the expected compression ratio and minimum pulse length for the case of an ideal velocitytilted beam with arbitrary distribution passing through a perfectly neutralizing plasma. However, such longitudinal emittance-dominated solutions do not include the finitesize gap effects, such as the reduction in tilt $f$, effective $T_{\|}$ increase, or increased longitudinal focusing aberration experienced by the head particles compared to the tail particles. The general derivation of the constant that takes into account the effective $T_{\|}$imparted onto the beam by the finite-size gap is not provided here. The effective longitudinal temperature of the beam exiting the gap is intimately related to the minimum pulse length when a nearly ideal waveform is employed, with smaller $T_{\|}$associated with higher compression ratios and shorter pulse lengths. Deviations from the ideal slope by the experimental waveform also increase the effective $T_{\|}$inferred by the measured pulse length. When such errors in the voltage waveform are large, the minimum pulse length is said to be waveform dominated, instead of emittance dominated. In reality, Eq. (18) is of limited value because the minimum achievable pulse length is actually a complex function of many realistic considerations, including partial neutralization effects.

\section{Dependence on initial pulse length and intended fractional tilt}

Since the voltage waveform of the induction module imparts the axial velocity tilt to the beam, it is inextricably linked to the amount of current compression achieved at the focal plane. Assume that the ideal waveform $V(t)$ can be accurately reproduced over the duration of the intended pulse length $t_{p}$, as in the smooth waveform. The two $V(t)$ waveform parameters that can be adjusted by the experimenter are the intended pulse length $t_{p}$ and the amount of desired fractional tilt $f$.

First, consider changes in $V(t)$ due to $t_{p}$, while holding $E_{0}=400 \mathrm{keV}$ and $f=0.5$ constant. The smooth waveforms for $t_{p}=100 \mathrm{~ns}$ to $500 \mathrm{~ns}$, in steps of $100 \mathrm{~ns}$, are displayed in Fig. 14. All of the waveforms considered here are $\pm 180 \mathrm{kV}$ symmetric, as opposed to the waveform in Fig. 1. The reason is that a plus-minus symmetric waveform takes the most advantage of the available voltage for a given limit, due to voltage hold-off considerations. The drift length $L_{d}$ increases linearly with $t_{p}$ for constant $E_{0}$ and $f$ [Eq. (6)], as does the amount of focusing aberration [Eqs. (12) and (13)] and the minimum achievable pulse length [Eq. (18)]. The competing effect is the increasing amount of longitudinal compression, since the compression of longer initial $t_{p}$ results in larger amounts of total current at the focal plane, assuming charge conservation $\left[I^{f} / I_{0}=\left(q^{f} / t_{p}^{f}\right)\left(q_{0} / t_{p}^{0}\right)^{-1}=t_{p}^{0} / t_{p}^{f}\right.$ for $\left.q^{f}=q_{0}\right]$. However, the effect plateaus due to the increased longitudinal focusing aberration associated with longer $L_{d}$. Therefore, careful assessment of the desired amount of compression compared to the desired minimum pulse length at focus and available plasma column for experiments is required.

The simulation results for current compression of an $E_{0}=400 \mathrm{keV}$ and $T_{\|}=0.2 \mathrm{eV} K^{+}$ion beam using the color-coded smooth waveforms of Fig. 14 across the $3 \mathrm{~cm}$ gap are shown in Fig. 15. The actual initial $t_{p}$ are longer than the prescribed lengths intended to undergo compression. The extra voltage in the smooth waveform acts on the extra initial $t_{p}$ to increase the compression ratio, tilt $f$, and $t_{\mathrm{FWHM}}$ (slightly) achieved at the focal plane compared to the sharp waveform case. The compression dependence on

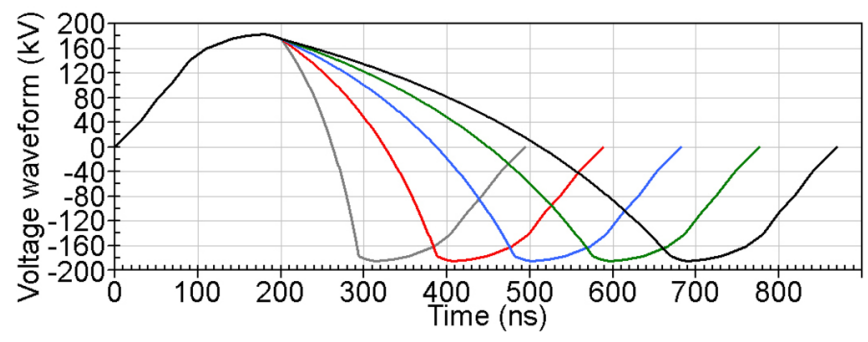

FIG. 14. (Color) Smooth waveforms $V(t)$ for $E_{0}=400 \mathrm{keV}$, $f=0.5$, and $t_{p}=100 \mathrm{~ns}$ (gray), $t_{p}=200 \mathrm{~ns}$ (red), $t_{p}=$ $300 \mathrm{~ns}$ (blue), $t_{p}=400 \mathrm{~ns}$ (green), and $t_{p}=500 \mathrm{~ns}$ (black). 


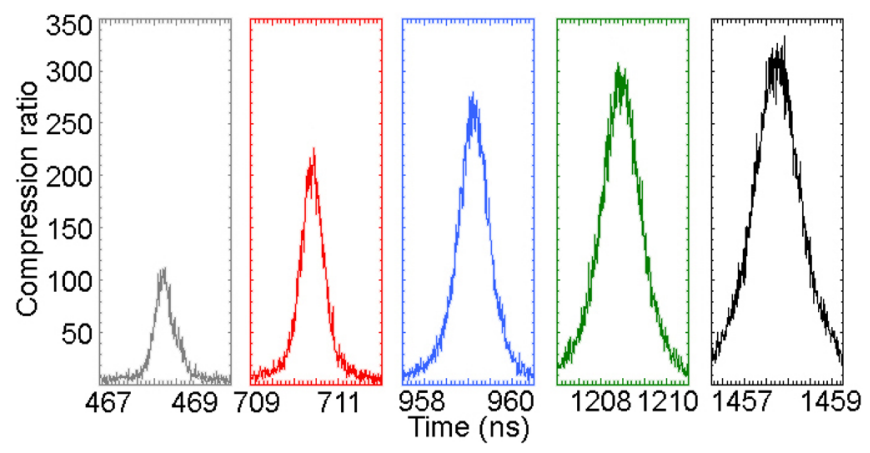

FIG. 15. (Color) Compression ratios $I_{b}(t) / I_{0}$ in the laboratory frame at the focal planes using the color-coded smooth waveforms of Fig. 14 across the finite-size gap for $T_{\|}=0.2 \mathrm{eV}, f=$ 0.5 , and $t_{p}=100 \mathrm{~ns}$ (gray), $t_{p}=200 \mathrm{~ns}$ (red), $t_{p}=300 \mathrm{~ns}$ (blue), $t_{p}=400 \mathrm{~ns}$ (green), and $t_{p}=500 \mathrm{~ns}$ (black).

initial pulse length at the focal plane is provided in Table II in terms of the compression ratio, full-width, halfmaximum pulse length, drift length, and the spatial offset between the focal lengths for the finite-size and infinitely thin gaps $\left(\Delta L_{d}\right)$. The data in Table II correspond to the results in Fig. 15.

The compression ratio increases as $\sim t_{p}^{0.8}$ for shorter initial pulse lengths, but as $\sim t_{p}^{0.3}$ for longer initial pulse lengths. The minimum $t_{\mathrm{FWHM}}$ increases as $\sim t_{p}^{0.3}$ for shorter initial pulse lengths, but as $\sim t_{p}^{1.05}$ for longer initial pulse lengths, in agreement with the linear dependence of Eq. (18). Recall that Eq. (18) underestimates the pulse length at focus by an approximately constant $20 \%$ for $t_{p} \geq$ $300 \mathrm{~ns}$, due to the $45 \%$ extra effective $T_{\|}$given to the beam by the gap effects. Although, as $t_{p}$ decreases to $200 \mathrm{~ns}$ and $100 \mathrm{~ns}$, the underestimation of $t_{\mathrm{FWHM}}$ by Eq. (18) increases to $36 \%$ and $62 \%$, respectively, since the initial $L_{b}$ and $t_{p}$ each become comparable to the effective gap length and transit time, respectively. The failure of Eq. (18) for short $t_{p}$ is expected, since its derivation assumed an infinitely thin gap relative to the initial $L_{b}$. Essentially, beam particles sample large amounts of the $V(t)$ if the gap is not thin enough, resulting in a reduced effective $f$ ( $v_{h}$ too large and $v_{t}$ too small) that is a function of initial $t_{p}$. Although none of the simulations recover the intended $f=0.5$, longer initial $t_{p}$ achieve greater $f$, but at the cost of more longi-

TABLE II. Compression dependence on initial pulse length $t_{p}$ for $E_{0}=400 \mathrm{keV}, T_{\|}=0.2 \mathrm{eV}$, and an intended $f=0.5 \mathrm{using}$ the smooth waveform across the $3 \mathrm{~cm}$ long gap.

\begin{tabular}{lcccc}
\hline \hline$t_{p}$ & $I_{b}^{\max } / I_{0}$ & $t_{\mathrm{FWHM}}$ & $L_{d}[$ Eq. (6)] & $\Delta L_{d}$ \\
\hline $100 \mathrm{~ns}$ & 120 & $0.5 \mathrm{~ns}$ & $26.2 \mathrm{~cm}$ & $+2.65 \mathrm{~cm}$ \\
$200 \mathrm{~ns}$ & 220 & $0.6 \mathrm{~ns}$ & $52.5 \mathrm{~cm}$ & $+1.45 \mathrm{~cm}$ \\
$300 \mathrm{~ns}$ & 280 & $0.7 \mathrm{~ns}$ & $78.7 \mathrm{~cm}$ & $+1.25 \mathrm{~cm}$ \\
$400 \mathrm{~ns}$ & 310 & $0.9 \mathrm{~ns}$ & $104.9 \mathrm{~cm}$ & $+1.15 \mathrm{~cm}$ \\
$500 \mathrm{~ns}$ & 325 & $1.2 \mathrm{~ns}$ & $131.2 \mathrm{~cm}$ & $+0.95 \mathrm{~cm}$ \\
\hline \hline
\end{tabular}

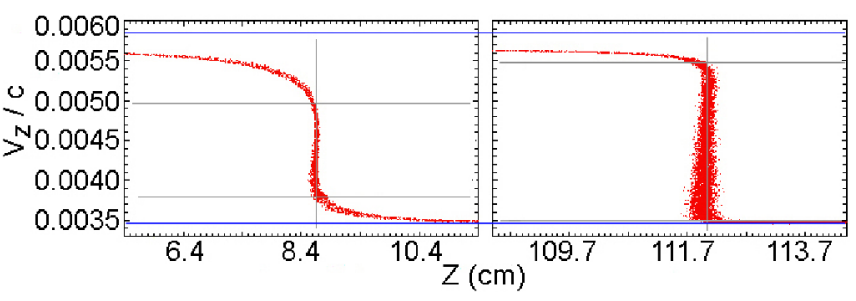

FIG. 16. (Color) $\left\{z, v_{z}\right\}$ phase space near the focal planes for the $t_{p}=100 \mathrm{~ns}$ (left) and $t_{p}=500 \mathrm{~ns}$ (right) cases shown in Fig. 15 and Table II. Blue and gray horizontal lines demarcate the expected $v_{h}$ and $v_{t}$ for $f=0.5$, and show the minimum $v_{h}$ and maximum $v_{t}$ achieved by the beam, respectively.

tudinal focusing aberration from $T_{\|}$over the greater $L_{d}$. Figure 16 illustrates both phenomena graphically. The actual $f$ achieved for $t_{p}=100 \mathrm{~ns}$ and $t_{p}=500 \mathrm{~ns}$ are $f \sim$ 0.25 and $f \sim 0.43$, respectively. The compression ratio for the $t_{p}=300 \mathrm{~ns}$ case is reduced to 280 (from 320 previously), and corresponds to the same percentage reduction in waveform voltage late in time ( $-225 \mathrm{kV}$ to $-180 \mathrm{kV})$.

The last column of Table II shows that, as the initial pulse length increases relative to the effective gap transit time, the spatial (and also temporal) offset between the focal lengths for the finite-size and infinitely thin gaps decreases $\left(\sim t_{p}^{-0.6}\right)$. Therefore, the origin of the offset mentioned earlier between the two gap cases lies in the quantitative ratios of $L_{b}$ and $t_{p}$ to the effective gap length and transit time, respectively. Another interpretation would be that the finite-size gap changes the resulting tilt $f$ while decreasing the $v_{t}$ more than increasing the $v_{h}$, and therefore $L_{d}$ [refer to Eq. (3)] becomes larger compared to the case where the velocities are changed by the same amount.

Second, consider changes in $V(t)$ due to the intended tilt $f$, while holding $E_{0}=400 \mathrm{keV}$ and $t_{p}=300 \mathrm{~ns}$ constant. The smooth waveforms for $f=0.3$ to $f=0.7$, in steps of $f=0.1$, are displayed in Fig. 17. Now none of the waveforms are $\pm V^{\max } \mathrm{kV}$ symmetric, in order to capture the full voltage range for each of the $f$. The required time and drift length to the focal plane decrease as $\frac{1}{f}+\frac{1}{2}$ and $\frac{1}{f}-\frac{f}{4}$, respectively, with increasing $f$ for constant $E_{0}$ and $t_{p}$ [Eqs. (5) and (6)]. The amount of longitudinal focusing aberration [Eqs. (12) and (13)] and the minimum pulse

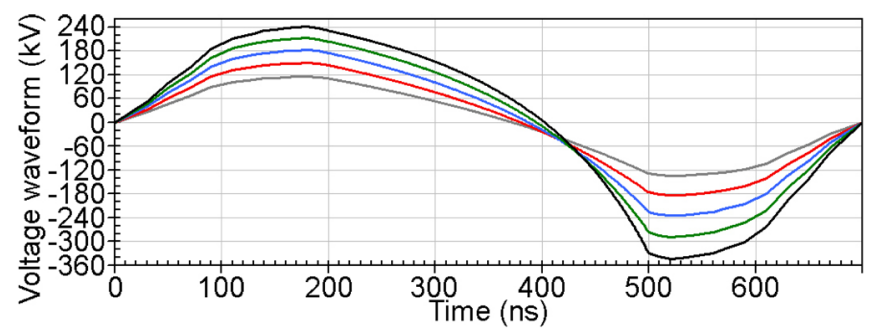

FIG. 17. (Color) Smooth voltage waveforms $V(t)$ for $E_{0}=$ $400 \mathrm{keV}, t_{p}=300 \mathrm{~ns}$, and $f=0.3$ (gray), $f=0.4$ (red), $f=$ 0.5 (blue), $f=0.6$ (green), and $f=0.7$ (black). 
length [Eq. (18)] decrease with increasing $f$, as well. Therefore, larger tilts are generally desired for increased levels of current compression and shorter pulse lengths. However, a careful assessment of the desired amount of compression compared to the increased transverse focusing aberration, associated with the energy tilt during transverse compression to a coincident focal plane, is required.

The simulation results for current compression of an $E_{0}=400 \mathrm{keV}$ and $T_{\|}=0.2 \mathrm{eV} K^{+}$ion beam using the color-coded smooth waveforms of Fig. 17 across the $3 \mathrm{~cm}$ gap are shown in Fig. 18. The actual initial $t_{p}$ are longer than the prescribed $t_{p}=300 \mathrm{~ns}$ intended to undergo compression. The compression dependence on the intended fractional tilt $f$ at the focal plane is provided in Table III in terms of the compression ratio, full-width, halfmaximum pulse length, drift length, and the spatial offset between the focal lengths for the finite-size and infinitely thin gap cases $\left(\Delta L_{d}\right)$. The data in Table III correspond to the results in Fig. 18.

The compression ratio increases as $\sim f^{0.82}$ for smaller intended $f(0.3 \leq f<0.5)$, but as $\sim f^{0.51}$ for larger intended $f(0.5 \leq f<0.7)$. The minimum $t_{\mathrm{FWHM}}$ decreases with increasing $f$ as $\sim f^{-1.23}$, in agreement with the $\sim f^{-1.25}$ dependence in Eq. (18). Although, the $t_{\mathrm{FWHM}}$ from Eq. (18) maintains its $20 \%$ underestimation at focus as a function of $f$, since the ratios of the initial $L_{b}$ and $t_{p}$ to the effective gap length and transit time, respectively, remain constant. The constant offset of Eq. (18) offers validation that the discrepancy is solely due to the relative size of the mentioned geometric parameters, and not the intended tilt $f$. As before, the intended fractional tilt is not achieved in any of the cases presented in Table III. The resulting relative decrease is approximately $20 \%$ for $t_{p}=$ $300 \mathrm{~ns}$ and independent of $f$. Recall that the compression ratio reduction and full-width, half-maximum pulse length increase due to the gap were also reported to be $20 \%$. Again, smaller tilts $f$ suffer more longitudinal focusing aberration from $T_{\|}$due to the greater $L_{d}$ required.

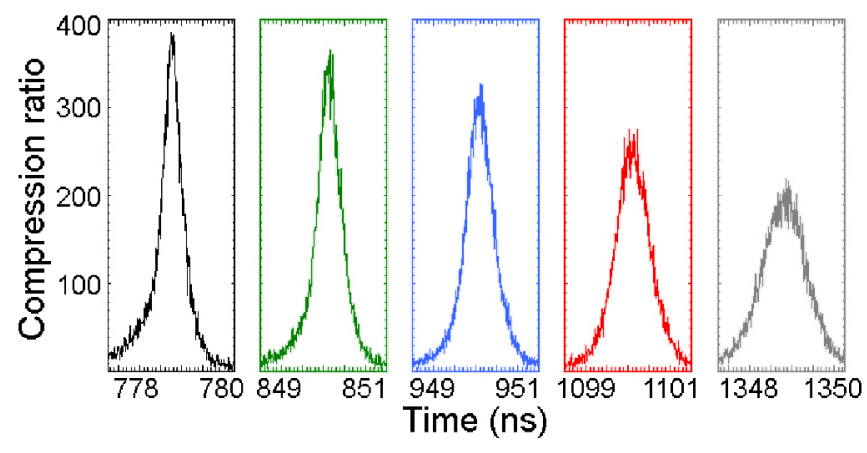

FIG. 18. (Color) Compression ratio $I_{b}(t) / I_{0}$ comparison using the color-coded smooth waveforms of Fig. 17 across the finitesize gap in the laboratory frame at the focal planes for $T_{\|}=$ $0.2 \mathrm{eV}, t_{p}=300 \mathrm{~ns}$, and $f=0.3$ (gray), $f=0.4$ (red), $f=0.5$ (blue), $f=0.6$ (green), and $f=0.7$ (black).
TABLE III. Compression dependence on the intended tilt $f$ for $E_{0}=400 \mathrm{keV}, T_{\|}=0.2 \mathrm{eV}$, and $t_{p}=300 \mathrm{~ns}$ using the smooth waveform across the $3 \mathrm{~cm}$ long gap.

\begin{tabular}{lcccc}
\hline \hline$f$ & $I_{b}^{\max } / I_{0}$ & $t_{\mathrm{FWHM}}$ & $L_{d}[$ Eq. (6)] & $\Delta L_{d}$ \\
\hline 0.3 & 210 & $1.4 \mathrm{~ns}$ & $136.8 \mathrm{~cm}$ & $+1.00 \mathrm{~cm}$ \\
0.4 & 260 & $1.0 \mathrm{~ns}$ & $100.7 \mathrm{~cm}$ & $+1.25 \mathrm{~cm}$ \\
0.5 & 320 & $0.7 \mathrm{~ns}$ & $78.7 \mathrm{~cm}$ & $+1.25 \mathrm{~cm}$ \\
0.6 & 360 & $0.6 \mathrm{~ns}$ & $63.7 \mathrm{~cm}$ & $+1.25 \mathrm{~cm}$ \\
0.7 & 380 & $0.5 \mathrm{~ns}$ & $52.6 \mathrm{~cm}$ & $+1.35 \mathrm{~cm}$ \\
\hline \hline
\end{tabular}

The last column of Table III shows that the spatial (and also temporal) offset between the focal lengths for the finite-size and infinitely thin gap cases is approximately independent of (or weakly dependent on) $f$, since the initial bunch and pulse lengths relative to the effective gap length and transit time across the gap, respectively, are approximately constant. In addition, the required time and drift length to the focal plane decrease approximately with $\sim f^{-0.81}$ and $\sim f^{-1.12}$ dependence, respectively, in agreement with Eqs. (5) and (6).

The results presented in this subsection indicate that an optimum drift length exists, for a given set of beam parameters, that balances the larger compression ratios achieved by longer initial $t_{p}$ and larger $f$ with the increased $t_{\mathrm{FWHM}}$ values of the former and decreased $t_{\mathrm{FWHM}}$ values of the latter. Longer initial $t_{p}$ are shown to increase $L_{d}$, as well as increase longitudinal focusing aberration, and shorter initial $t_{p}$ are shown to achieve smaller $f$ due to the finite-size gap. Also, larger tilts are shown to decrease $L_{d}$ and longitudinal focusing aberration, but will adversely affect the transverse focusing aberration. The amount of tilt achieved in the laboratory is limited by the magnetic flux of the induction module and voltage hold-off considerations in the gap.

\section{Dependence on energy and thermal distribution}

The last topic covered in this section is the dependence of current compression on the ion beam kinetic energy and distribution function. The derivation of the ideal $V(t)$ assumed a particular initial (and constant) beam energy $E_{0}$. Since the $V(t)$ slope is only ideal for one particular $E_{0}$, slight energy differences cause the beam to focus to different axial locations, with lower compression ratios and longer pulse lengths. Also, the longitudinal distribution is expected to affect compression, since longitudinal focusing aberration causes smearing of the focal plane in time and space. Then, the resulting current profile is a direct consequence of the distribution of particle energies, and both the average $E_{0}$ [or $\left.E_{0}(t)\right]$ and the distribution play a role. Various pulse shapes may therefore be realized at the target.

In the NDCX experiment, the accuracy of $E_{0}$ is approximately $\pm 5 \%$. In order to maximize the reliability of the 
longitudinal focusing capability, the shot-to-shot variation in directed beam energy and imposed waveform must be minimized. Also, the $E_{0}(t)$ over the microseconds of its long initial pulse length might generally not be constant, since it depends on the high-power diode technology employed. In practice, the injected $E_{0}(t)$ in the NDCX experiment may fluctuate on fast time scales (contributing to longitudinal emittance), as well as drift its mean energy by as much as $\pm 10 \%$ over a $t_{p}=6-10 \mu$ s pulse, or as much as $\pm 1 \%-2 \%$ over a window of $\sim 300 \mathrm{~ns}$. In order to quantify the effects on compression from these two types of energy variations, a set of simulations is carried out.

First, recall the compression simulation across the finitesize gap using a smooth waveform, with the parameters $E_{0}=400 \mathrm{keV}, t_{p}=300 \mathrm{~ns}$, and $f=0.5$, and an initialized Maxwellian temperature of $T_{\|}=0.2 \mathrm{eV}$ just upstream of the acceleration gap; the results are in Fig. 10, and the focal plane is located at $z=+59.5 \mathrm{~cm}$ and $t=$ 959 ns. The simulation is repeated 4 times, except the constant initial beam energy is changed to $E_{0}=380 \mathrm{keV}$ $(-5 \%), 390 \mathrm{keV}(-2.5 \%), 410 \mathrm{keV}(+2.5 \%)$, and $420 \mathrm{keV}(+5 \%)$, in order to represent the $\pm 5 \%$ accuracy of the $E_{0}$ in the NDCX experiments.

The current compression profiles through the originally intended focal plane for the four cases are shown in Fig. 19. The more energetic beams arrive first, followed by the less energetic beams in order. The two beam cases with larger $v_{0}$ have not reached their focal planes at this $z$ because their $L_{d}$ are longer. The opposite is true for the charge bunches with smaller $v_{0}$, and those two cases are already beyond their focal planes at this $z$.

In order to directly compare the amount of compression achieved by the different $E_{0}$ beams using the waveform intended for an $E_{0}=400 \mathrm{keV}$ beam, the current profiles through their respective focal planes are plotted as a function of absolute time on a linear scale in Fig. 20; the five plots are color coded to match those in Fig. 19. The compression dependence at the focal plane is provided in

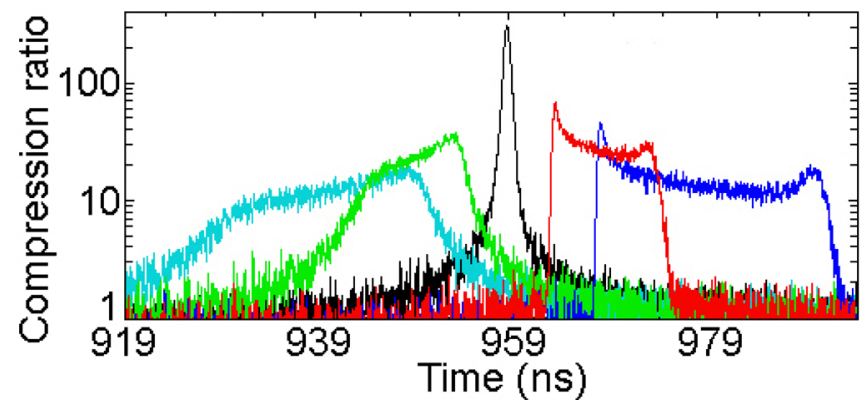

FIG. 19. (Color) Compression ratio $I_{b}(t) / I_{0}$ (log scale) comparison of $\pm 5 \%$ constant $E_{0}$ variations using the smooth waveform across the finite-size gap and $T_{\|}=0.2 \mathrm{eV}$ beams at $z=$ $+59.5 \mathrm{~cm}$ in the laboratory frame for $E_{0}=420 \mathrm{keV}$ (light blue), $E_{0}=410 \mathrm{keV}$ (green), $E_{0}=400 \mathrm{keV}$ (black), $E_{0}=$ $390 \mathrm{keV}$ (red), and $E_{0}=380 \mathrm{keV}$ (dark blue).

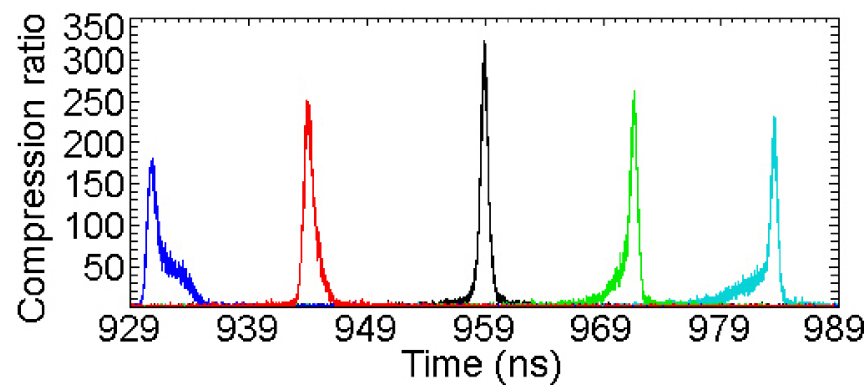

FIG. 20. (Color) Compression ratio $I_{b}(t) / I_{0}$ comparison in the laboratory frame for $\pm 5 \%$ constant $E_{0}$ variations using the smooth waveform across the finite-size gap and $T_{\|}=0.2 \mathrm{eV}$ beams for $E_{0}=420 \mathrm{keV}$ (light blue) at $z=+65.0 \mathrm{~cm}, E_{0}=$ $410 \mathrm{keV}$ (green) at $z=+62.3 \mathrm{~cm}, E_{0}=400 \mathrm{keV}$ (black) at $z=+59.5 \mathrm{~cm}, E_{0}=390 \mathrm{keV}$ (red) at $z=+56.4 \mathrm{~cm}$, and $E_{0}=380 \mathrm{keV}$ (dark blue) at $z=+53.6 \mathrm{~cm}$. The plots are color coded to the profiles of the same color in Fig. 19.

Table IV in terms of the compression ratio, full-width, halfmaximum pulse length, total time to focus, and the location of the focal plane in the PIC axial coordinates. The data in Table IV correspond to the results in Fig. 20.

Small $\pm 2.5 \%-5 \%$ discrepancies in $E_{0}$ from the intended beam energy for the particular $V(t)$ result in a range of approximately $20 \%-40 \%$ relative decrease, respectively, in the amount of compression measured at the different focal planes. Higher energies achieve shorter $t_{\mathrm{FWHM}}$ at longer $L_{d}$ and later in time. However, the $t_{\mathrm{FWHM}}$ value does not reflect the significant current pedestals formed by the energy inaccuracy, which are particularly virulent for the $\pm 5 \%$ cases in Fig. 20. Changes in beam energy by $\pm 10 \mathrm{keV}( \pm 2.5 \%)$ result in an average change of $\pm 2.8 \mathrm{~cm}$ in the focal plane location.

A particular $V(t)$ slope results in optimum compression only for a particular $E_{0}$ at the intended focal plane. Beam energy scans to align a diagnostic with a peak compression result, while holding $V(t)$ fixed, do not unambiguously locate the true optimum compression achievable for the waveform under consideration, as quantitatively shown in Fig. 20 and Table IV. Discernment of the actual optimum compression due to a particular waveform is possible by first fixing both $E_{0}$ and $V(t)$ and conducting an axial scan of

TABLE IV. Compression dependence on initial $E_{0}$ inaccuracy over a $\pm 5 \%$ range with $T_{\|}=0.2 \mathrm{eV}$ for $E_{0}=400 \mathrm{keV}, t_{p}=$ $300 \mathrm{~ns}$, and $f=0.5$ using the smooth waveform across the $3 \mathrm{~cm}$ long gap.

\begin{tabular}{lcccc}
\hline \hline$E_{0}$ & $I_{b}^{\max } / I_{0}$ & $t_{\mathrm{FWHM}}$ & $t^{\mathrm{foc}}$ & $z^{\mathrm{foc}}$ \\
\hline $380 \mathrm{keV}$ & 180 & $0.9 \mathrm{~ns}$ & $930.9 \mathrm{~ns}$ & $+53.6 \mathrm{~cm}$ \\
$390 \mathrm{keV}$ & 250 & $0.8 \mathrm{~ns}$ & $944.1 \mathrm{~ns}$ & $+56.4 \mathrm{~cm}$ \\
$400 \mathrm{keV}$ & 320 & $0.7 \mathrm{~ns}$ & $959.0 \mathrm{~ns}$ & $+59.5 \mathrm{~cm}$ \\
$410 \mathrm{keV}$ & 260 & $0.7 \mathrm{~ns}$ & $971.8 \mathrm{~ns}$ & $+62.3 \mathrm{~cm}$ \\
$420 \mathrm{keV}$ & 230 & $0.6 \mathrm{~ns}$ & $983.7 \mathrm{~ns}$ & $+65.0 \mathrm{~cm}$ \\
\hline \hline
\end{tabular}


the profiles in the focal plane vicinity with a movable diagnostic. Once a set of ratios $I_{b}(t) / I_{0}$ at multiple axial locations before and after the focal plane is measured, such as many of those presented throughout this paper, the repetition of such a set of measurements for various $E_{0}$ with $V(t)$ fixed will result in the conclusive determination of the achievable optimum compression for the given $V(t)$. The peak compression profiles from each set of measurements for various $E_{0}$ would look similar to Fig. 20 when plotted together. Without an axial scan by the diagnostic for a range of $E_{0}$, any of the measured maximum compression ratios portrayed in Fig. 20 may be mistaken for the "optimum" compression ratio when, in fact, only one of them may be so called (the black one).

Second, the $E_{0}$ of the injected beam might not be generally uniform. In the NDCX experiment, the beam energy may fluctuate on fast time scales, as well as drift its mean energy by as much as $\pm 1 \%-2 \%$ over a window of $\sim 300 \mathrm{~ns}$. In order to quantify the effects of an average energy drift as the beam enters the acceleration gap, two additional similar simulations are carried out. The first allows the $E_{0}(t)$ of the $400 \mathrm{keV}$ beam to rise linearly with time over the pertinent $t_{p}=300 \mathrm{~ns}$ of the waveform to $E_{0}=408 \mathrm{keV}(+2 \%)$; the second allows the $E_{0}(t)$ of the same beam to sink linearly with time over the pertinent $t_{p}=300$ ns to $E_{0}=392 \mathrm{keV}(-2 \%)$.

In order to compare the amount of compression achieved by the beams with increasing and decreasing $E_{0}(t)$ using the waveform intended for a $E_{0}=400 \mathrm{keV}$ beam, the current profiles through their respective focal planes are plotted as a function of absolute time on a linear scale in Fig. 21. The focal plane of the original $E_{0}=400 \mathrm{keV}$ simulation is located at $z=+59.5 \mathrm{~cm}$ and $t=959 \mathrm{~ns}$. Contrary to the previous simulation, the slightly more energetic beam arrives at its focal plane first, followed by the nominal and slightly less energetic beams, in order. The compression dependence at the focal plane on initial $E_{0}$

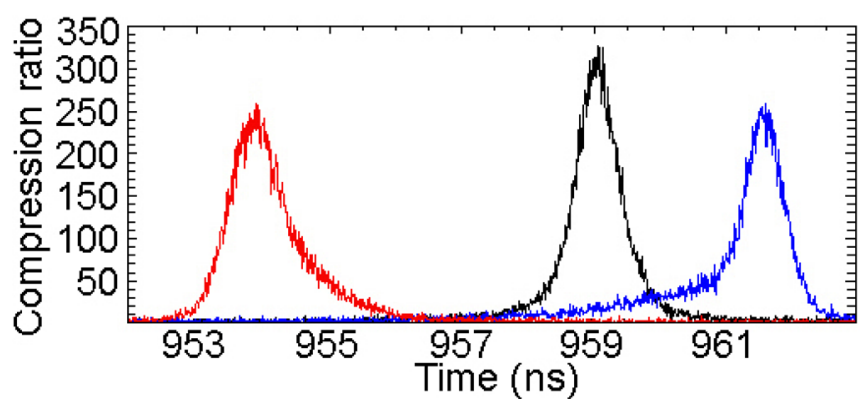

FIG. 21. (Color) Compression ratio $I_{b}(t) / I_{0}$ comparison in the laboratory frame of initial $E_{0}$ with linear increase or decrease by $\pm 2 \%$ over the pertinent $t_{p}=300 \mathrm{~ns}$, using the smooth waveform across the finite-size gap, for $T_{\|}=0.2 \mathrm{eV}$ beams with rising $E_{0}(t)=400-408 \mathrm{keV}$ (red) at $z=+59.3 \mathrm{~cm}$, constant $E_{0}(t)=400 \mathrm{keV}$ (black) at $z=+59.5 \mathrm{~cm}$, and sinking $E_{0}(t)=$ 400-392 keV (dark blue) at $z=+59.3 \mathrm{~cm}$.
TABLE V. Compression dependence on initial linear increase or decrease in $E_{0}(t)$ by $\pm 2 \%$ with $T_{\|}=0.2 \mathrm{eV}$ for $E_{0}=$ $400 \mathrm{keV}, t_{p}=300 \mathrm{~ns}$, and $f=0.5$ using the smooth waveform across the $3 \mathrm{~cm}$ long gap.

\begin{tabular}{lcccc}
\hline \hline$E_{0}$ & $I_{b}^{\max } / I_{0}$ & $t_{\mathrm{FWHM}}$ & $t^{\mathrm{foc}}$ & $z^{\text {foc }}$ \\
\hline $400-392 \mathrm{keV}$ & 250 & $0.8 \mathrm{~ns}$ & $961.5 \mathrm{~ns}$ & $+59.3 \mathrm{~cm}$ \\
$400 \mathrm{keV}$ & 320 & $0.7 \mathrm{~ns}$ & $959.0 \mathrm{~ns}$ & $+59.5 \mathrm{~cm}$ \\
$400-408 \mathrm{keV}$ & 250 & $0.8 \mathrm{~ns}$ & $953.9 \mathrm{~ns}$ & $+59.3 \mathrm{~cm}$ \\
\hline \hline
\end{tabular}

with linear increase or decrease by $\pm 2 \%$ over the $t_{p}=$ $300 \mathrm{~ns}$ window is provided in Table $\mathrm{V}$ in terms of the compression ratio, full-width, half-maximum pulse length, total time to focus, and the location of the focal plane in the PIC axial coordinates. The data in Table V correspond to the results in Fig. 21.

Small $\pm 2 \%$ increases or decreases in $E_{0}(t)$ from the intended energy over the $t_{p}=300 \mathrm{~ns}$ pulse length for the particular $V(t)$ result in an approximate $22 \%$ relative decrease in the amount of achieved compression. Both types of initial energy change achieve longer $t_{\mathrm{FWHM}}$ at a focal plane upstream of the nominal case, for the parameters considered here; although, the values of $t_{\mathrm{FWHM}}$ do not reflect the significant pedestals formed by the energy drifts, which are particularly prevalent in both cases in Fig. 21. Contrary to intuition, both types of temporal change in $E_{0}(t)$ result in the formation of a focal plane at the same axial location, approximately $0.2 \mathrm{~cm}$ upstream of the nominal location, for the parameters considered here. The reason for the departure from the expected, such as the shorter (longer) drift length and time to focus for the faster (slower) beam, is that the linear temporal increase (decrease) in $E_{0}(t)$ is effectively a compressing (decompressing) initial velocity tilt. Therefore, the beam whose energy increases (decreases) as a function of time entering the gap acquires a slightly larger (smaller) total tilt than nominal. Consequently, the relative $L_{d}$ of the increasing (decreasing) energy case is slightly shorter (longer) than intended. In both cases, the peak compression ratio is relatively decreased compared to the beam with the proper $E_{0}$ for the $V(t)$.

Unintentional experimental $E_{0}(t)$ increases or decreases, whether constant in time or not, will not reach the optimum compression achievable compared to the appropriate constant $E_{0}$ case for the specific $V(t)$. This is quantitatively illustrated in Figs. 20 and 21 and Tables IV and V. In summary, if a beam enters the gap with an $E_{0}(t)$ too high/low (or increasing/decreasing in time) by a few percent, one of the suboptimal "maximum" compression ratios may be measured and mistaken for the actual optimum ratio. Beams with constant $E_{0}$ a few percent larger (smaller) than the appropriate $E_{0}$, or with an $E_{0}(t)$ profile rising (sinking) by a few percent over the initial $t_{p}$, result in the deformation of the vertical $\left\{z, v_{z}\right\}$ phase space at focus into one slightly curved forward (backward), rather than 
entirely vertical. Both types of change reduce compression by smearing the focal plane in a manner similar to longitudinal focusing aberration.

The type of beam distribution that characterizes the longitudinal temperature affects the shape of the current profiles, and therefore the associated compression. The original simulation is revisited one last time in order to discern the effect on longitudinal focus (at $z=+59.5 \mathrm{~cm}$ and $t=959 \mathrm{~ns}$ ) when the $T_{\|}=0.2 \mathrm{eV}$ Maxwellian distribution is changed to a $T_{\|}=0.6 \mathrm{eV}$ Waterbag distribution. Recall that the Waterbag distribution is a uniform-density and equally populated distribution between $v_{0}-v_{\text {th }}$ and $v_{0}+v_{\text {th }}$, and the two distributions satisfy $v_{\mathrm{th}}^{W}=\sqrt{3} v_{\mathrm{th}}^{M}$ for $T_{M}=T_{W}$. However, in order to equally compare the shape of the two distributions, the width of the Waterbag distribution must be equal to the full $3 v_{\mathrm{th}}^{M}(3 \sigma=99.73 \%)$ Maxwellian distribution, so $v_{\mathrm{th}}^{W}=3 v_{\mathrm{th}}^{M}$ and $T_{W}=3 T_{M}$ are employed here.

The current profiles for the Maxwellian and Waterbag distributions near the shared focal plane $(z=+59.5 \mathrm{~cm})$ are plotted as a function of absolute time on a linear scale in Fig. 22. At $t=959 \mathrm{~ns}$, the current profile in the Maxwellian case peaks at a compression ratio of 320, whereas the current in the Waterbag case is near a local minimum compression ratio of 150 . Compression ratios closer to the Maxwellian case maximum in the Waterbag case (300-320) are found just upstream and downstream from the intended focal plane at later and earlier times, respectively. The $t_{\mathrm{FWHM}}$ are similar, but the Maxwellian case maintains higher compression ratios near the profiles' centers due to the increased velocity space population densities near the desired $v_{z}$ values for the $V(t)$ compared to the Waterbag case, whose velocity space population densities are equidistant about the same desired $v_{z}$ values.

The bell-shaped normal distribution in the Maxwellian case achieves more compression and therefore shorter values of $t_{\text {FWHM }}$ times. The bell shapes in the

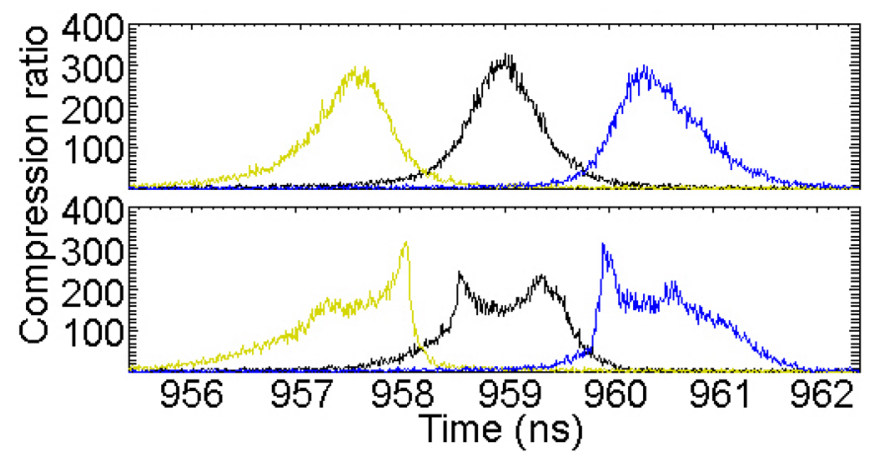

FIG. 22. (Color) Compression ratio $I_{b}(t) / I_{0}$ comparison for the smooth waveform across the finite-size gap in the laboratory frame at $z=+59.3 \mathrm{~cm}$ (yellow), at the focal plane $z=$ $+59.5 \mathrm{~cm}$ (black), and at $z=+59.7 \mathrm{~cm}$ (blue) for beams initialized with a $T_{\|}=0.2 \mathrm{eV}$ Maxwellian (top profiles) and $T_{\|}=$ $0.6 \mathrm{eV}$ Waterbag distribution (bottom profiles).
Maxwellian case are replaced with shapes approximating squares in the Waterbag case. The sharp peaks in current late (early) in time for individual profiles upstream (downstream) of the intended focus are due to the sharp velocity space boundaries of the Waterbag distribution; the more diffuse Maxwellian case does not have the same features. The sharp Waterbag velocity space boundaries for the faster tail particles contribute to relatively larger peaks in current because of the decreased levels of longitudinal focusing aberration suffered by the tail compared to the head. Therefore, the relatively increased current peaks arrive later (earlier) in time for profiles upstream (downstream) of the axial focal plane. In practice, intense beam distributions are closer to Maxwellian because of their creation from an emitter maintained at a certain temperature in the NDCX device. However, the blue Waterbag profile in Fig. 22 suggests that, if an ion beam with a Waterbag distribution could be used for compression experiments, preheat of targets could be reduced because the prepulse current pedestal is lower compared to the Maxwellian analog, and the elapsed time required by the Waterbag profile to increase from low to peak current is shorter than the same required by the Maxwellian distribution.

\section{SIMULTANEOUS TRANSVERSE AND LONGITUDINAL ION BEAM FOCUSING}

At the present time, the compression of ion beams in the NDCX experiment has been separately demonstrated in the transverse and longitudinal directions. However, simultaneous focusing to a common focal plane is desired in order to maximize the intensity on the target [18]. The goal for upcoming experiments is to demonstrate the feasibility and study the physical limitations of simultaneous transverse and longitudinal compression of an intense charge bunch $[7,15,24]$.

\section{A. Transverse defocusing effect}

As mentioned in Sec. III A, the process of imposing an axial velocity tilt for the purpose of longitudinal compression contributes a time-dependent net divergence to the otherwise radially converging trajectories of the ions $[18,21]$, as well as imparts velocity spread akin to an effective longitudinal temperature $T_{\|}$onto the beam. The time-dependent nature of the $V(t)$, combined with the sizable transit time of the ion particles across the gap, imply an imbalance of integrated $E_{r}(r, z, t)$ experienced by each particle and provides a time-dependent radial defocusing force to both the head and tail of the beam, as illustrated in Fig. 23.

A 2D $\{r, z\}$ PIC simulation of transverse focusing without an axial velocity tilt is compared to the same with an applied axial velocity tilt. An $E_{0}=300 \mathrm{keV} K^{+}$beam with an initial radius $r_{b}=2 \mathrm{~cm}$ and $I_{0}=18 \mathrm{~mA}$ of 


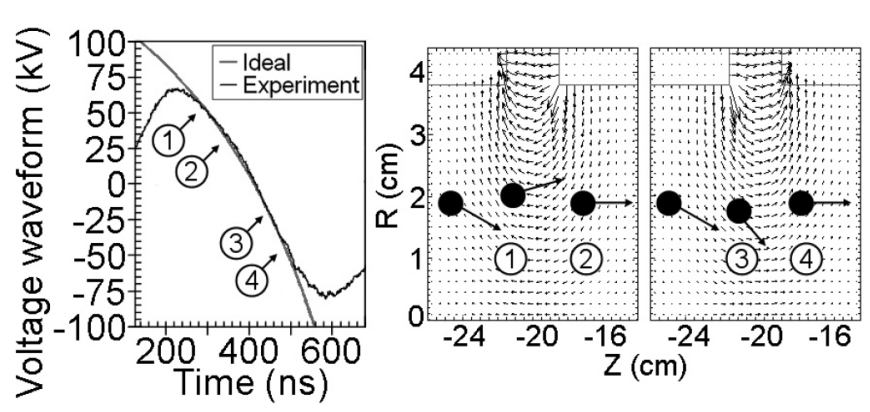

FIG. 23. (Color) Illustration of the radial defocusing effect of the acceleration gap. For $d V(t) / d t<0$, particles crossing the gap early in time [when $V(t)>0$, shown as 1 and 2] sample more integrated $+E_{r}$ at the entrance compared to the exit, whereas particles crossing the gap late in time [when $V(t)<0$, shown as 3 and 4] sample more integrated $+E_{r}$ at the exit compared to the entrance. In the center and right frames, electric field vectors are plotted and the black arrows indicating particle velocity vectors are exaggerated.

beam current is initialized with a Maxwellian $T_{\perp}=T_{\|}=$ $0.2 \mathrm{eV}$ distribution and a convergence angle of $\Delta \theta_{r}=$ $-15.4 \mathrm{mrad}$ at the injection plane $z=-30 \mathrm{~cm}$, just upstream of the gap. The initial $t_{p}=700 \mathrm{~ns}$ and, in the case with the velocity tilt, the $V(t)$ waveform imposes an approximate $f \sim 0.1$ tilt to the beam. The background plasma is sufficiently dense and cold to ensure good neutralization throughout the drift length.

Particle phase space plots with and without the velocity tilt are displayed in Fig. 24 after the beam passes the acceleration gap and enters the plasma $(t=1000 \mathrm{~ns})$. The difference in trajectories due to acceleration gap dynamics is clear. A hump shape in $\{r, z\}$ space around the region of the axially compressing charge bunch appears due to the radial defocusing effect, and the $r_{b}(t)$ at focus is increased considerably $(\sim 2 \mathrm{~cm})$ compared to the case without a tilt $(\sim 0.2 \mathrm{~cm})$. The consequence of the timedependent radial defocusing effect on current density compression is striking, because of the $r_{b}(t)^{-2}$ dependence on $J_{z}$. Early longitudinal compression experiments substantiated the existence of the effect [11], and agreed with simulations.

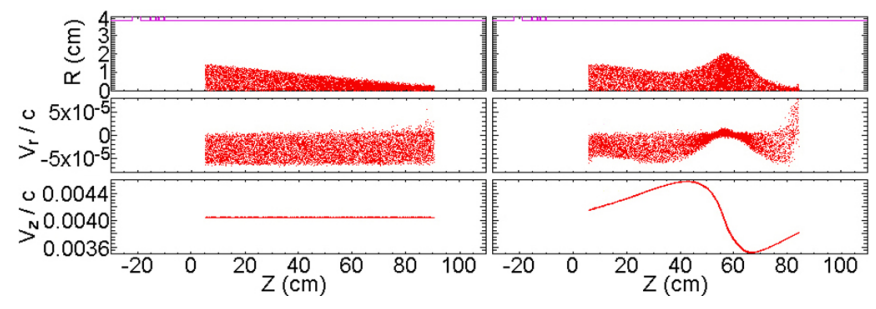

FIG. 24. (Color) Beam phase space comparison between transverse focusing without (left) and with (right) an axial velocity tilt at $t=1000 \mathrm{~ns}$, shown as particle plots in $\{r, z\}$ (top), $\left\{z, v_{r}\right\}$ (middle), and $\left\{z, v_{z}\right\}$ (bottom) coordinates. The radial defocusing effect imparts excess divergence to the longitudinally compressing portion of the beam in a time-dependent manner.

\section{B. Final-focus solenoid for transverse focusing}

One method for "refocusing" the defocused beam in order to achieve simultaneous compression to a submillimeter $r_{b}$ at the focal plane involves the use of a strong final-focus solenoid [7,18]. The use of a final-focus solenoid near the end of the drift length controls the effective focal length of the transverse compression, and therefore helps minimize the amount of plasma required throughout the system. A strong axial magnetic field has been shown to reduce magnetic filamentation, particularly at high beam densities, since plasma electrons are magnetized and constrained to move along field lines [25]. Two other methods under active research are the "over-focusing" technique [18], which only offsets the defocusing effect in an average way, and corrective time-dependent magnetic focusing, the technology for which is currently unavailable.

An energy-tilted beam entering a final-focus solenoid for transverse compression results in aberration of the transverse focal plane $[7,24]$. For a static $\mathbf{B}^{\mathrm{sol}}(r, z)$, the lower-energy head (higher-energy tail) of the tilted beam transversely focuses earlier (later) in space and time; the axial placement of the solenoid must be precisely positioned in order to ensure the optimal amount of simultaneous compression. A smaller tilt $f$ alleviates some of the transverse focusing aberration, but at the cost of reducing the longitudinal compression. The $\mathbf{B}$ field of a finite length and radius solenoid is modeled in the LSP code by using a power-series expansion of the radial coordinate, which involves only the on-axis $r=0$ field and its derivatives [26]. The PIC simulations employ a strong $150 \mathrm{kG}$ finalfocus solenoid of length $l^{\mathrm{sol}}=20 \mathrm{~cm}$ and radius $r^{\mathrm{sol}}=$ $3.8 \mathrm{~cm}$, centered at $z=+52.6 \mathrm{~cm}$, as illustrated in Fig. 25 . In general, the location of the transverse focal plane (and the amount of focusing aberration it suffers) depends on the length and strength of the solenoid, as well as the $r_{b}(t)$ and velocity space distribution of the beam as it enters the solenoid.

In order to quantify the effects arising from the solenoid rather than those from the gap, the case from Sec. III B of an ideally tilted beam using the sharp waveform across an

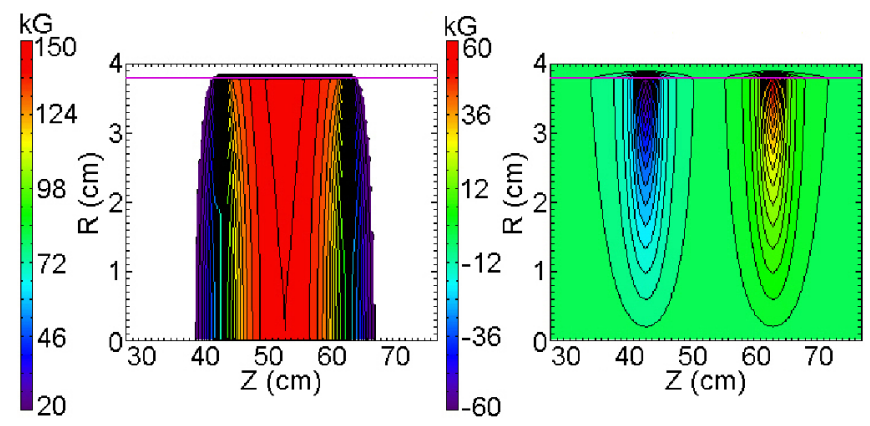

FIG. 25. (Color) $\{r, z\}$ isocontours of axial $B_{z}$ (left) and radial $B_{r}$ (right) field magnitudes from a $150 \mathrm{kG}$ solenoid of length $l^{\text {sol }}=$ $20 \mathrm{~cm}$ and radius $r^{\mathrm{sol}}=3.8 \mathrm{~cm}$, centered at $z=+52.6 \mathrm{~cm}$. 
infinitely thin gap, with initial parameters $E_{0}=400 \mathrm{keV}$, $t_{p}=300 \mathrm{~ns}, \quad f=0.5$, and Maxwellian $T_{\perp}=T_{\|}=$ $0.2 \mathrm{eV}$, is revisited with the addition of the $150 \mathrm{kG}$ solenoid. The original simulation resulted in a compression ratio of $I_{b}^{\max } / I_{0}=400$ with $t_{\mathrm{FWHM}}=0.6 \mathrm{~ns}$ at the focal plane $z=+58.25 \mathrm{~cm}$ at time $t=950 \mathrm{~ns}$. Here, $\left|B_{z}\right| \sim$ $140 \mathrm{kG}$ at the intended focal plane, although the focal plane can also be chosen to lie beyond the solenoid (depending on its length and strength). The beams are given initial radii of either $r_{b}=2 \mathrm{~cm}$ or $r_{b}=1 \mathrm{~cm}$ at $z=$ $-30 \mathrm{~cm}$, without convergence angles, and an initial current of $80 \mathrm{~mA}$, which approximately represent the peak parameters available in the NDCX device. The plasma is assumed to be sufficiently dense and cold in order to provide neutralization of the beam throughout the drift length.

The less energetic head of the compressing charge bunch arrives at the final-focus solenoid first. Strong $-B_{r}(r, z)$ fields are encountered, which rotate the beam via the Lorentz force, $-F_{\theta}=+q\left[\left(+v_{z}\right) \times\left(-B_{r}\right)\right]=-m_{i}$ $(\mathrm{d} \mathbf{v} / \mathrm{d} t)_{\theta}$. As found in the acceleration gap, the transverse and longitudinal dynamics are coupled, since the $+v_{z}$ particle motion increases the $-v_{\theta}$ rotation and leads to radial focusing, $\quad-F_{r}=+q\left[\left(-v_{\theta}\right) \times\left(+B_{z}\right)\right]=-m_{i}$ $(\mathrm{d} \mathbf{v} / \mathrm{d} t)_{r}$. In addition, the $-v_{\theta}$ is also the cause of axial deceleration via $-F_{z}=+q\left[\left(-v_{\theta}\right) \times\left(-B_{r}\right)\right]=-m_{i}$ $(\mathrm{d} \mathbf{v} / \mathrm{d} t)_{z}$, a second-order effect. Beam particles at larger radii entering the solenoid encounter stronger $-B_{r}(r, z)$ fields and acquire more $-v_{\theta}$, and subsequently more $-F_{r}$ focusing (and $-F_{z}$ deceleration), than those closer to the axis. All of the aforementioned velocities are functions of space and time due to the specific $\mathbf{B}^{\text {sol }}(r, z)$.

In addition to the cases involving beams of constant current density with initial radii of either $r_{b}=2 \mathrm{~cm}$ or $r_{b}=1 \mathrm{~cm}$ over the initial $t_{p}=300 \mathrm{~ns}$, two cases of radially constant but temporally decreasing current density $\left[J_{z}(t) \sim r_{b}(t)^{-2}\right]$ with linearly increasing $r_{b}(t)$ from $r_{b}=$ $0.1 \mathrm{~cm}$ to $r_{b}=2$ or $1 \mathrm{~cm}$ over the initial pulse length are considered. The $I_{0}$ through the $z=-30 \mathrm{~cm}$ plane is a constant $80 \mathrm{~mA}$ in all cases. It should be noted that, although the longitudinal emittances are the same in all the cases, the transverse emittances are not because of their dependence on the beam radius $r_{b}$ with constant $T_{\perp}$. The time-averaged transverse emittances (over the duration of the $t_{p}=300 \mathrm{~ns}$ ) are comparable, however. For the transverse emittances to be identical, time-dependent $T_{\perp}$ would need to be imposed on the injected beams according to Eq. (9). For the nonconstant $J_{z}(t)$ cases, the optimum positioning of the solenoid is moved by $\Delta z=+4 \mathrm{~cm}$ to $z=+56.6 \mathrm{~cm}$.

The reasoning behind the investigation of the cases involving a decreasing initial $J_{z}(t)$ with linearly increasing initial $r_{b}(t)$ is as follows. Slower head particles will normally transversely focus earlier than faster tail particles, contributing to transverse focusing aberration approxi- mately proportional to $r$ for a constant $r_{b}$. In order for the slower (faster) charge bunch head (tail) to transversely focus in a longer (shorter) axial distance and amount of time, they must enter the weaker (stronger) static $-B_{r}(r, z)$ fields at smaller (larger) radii, and thereby decrease (increase) the relative amount of $-v_{\theta}$ rotation and radial focusing force $-F_{r}$ they experience. Since the velocity tilt is nearly linear, and $-B_{r}$ increases approximately linearly with $r$ for $r<r^{\text {sol }}$, a charge bunch whose $r_{b}(t)$ increases linearly from head to tail along the pulse length as it enters a final-focus solenoid should minimize the associated focusing aberration in both directions.

The $\left\{z, v_{z}\right\}$ beam phase space for the four cases is shown in Fig. 26 at their respective focal planes. The original longitudinal focal plane was at $z=+58.25 \mathrm{~cm}$ without the solenoid, about $0.4-1.15 \mathrm{~cm}$ downstream of those in the cases presented here (due to the net $-v_{z}$ second-order effects shortening the drift lengths). The original longitudinal compression results are recovered in the case involving the linearly increasing $r_{b}$ to $1 \mathrm{~cm}$.

When the finite-size gap effects are included, the exiting $r_{b}(t)$ profile can be approximated as having a linear increase (decrease) over the first (second) half of the initial $t_{p}$, due to the transverse defocusing effect [refer to Fig. 24]. Such a triangular (or, parabolic because of the velocity tilt) pulse shape is simulated with linearly increasing $r_{b}(t)$ from $r_{b}=0.1 \mathrm{~cm}$ to $r_{b}=2 \mathrm{~cm}$ or $1 \mathrm{~cm}$ over the first half and linearly decreasing $r_{b}(t)$ from $r_{b}=2 \mathrm{~cm}$ or $1 \mathrm{~cm}$ to $r_{b}=$ $0.1 \mathrm{~cm}$ over the second half duration of the initial $t_{p}$ [with radially constant $J_{z}(t) \sim r_{b}(t)^{-2}$ temporal variation to ensure a constant $I_{0}=80 \mathrm{~mA}$ ]. Since the first half of the pulse has the desirable linear $r_{b}(t)$ increase, the triangular case is expected to return intermediate compression results between the constant and linearly increasing $r_{b}(t)$ cases.

Radial profiles of cumulative energy deposition $E_{\mathrm{dep}}(r)$ for the six cases under discussion are displayed in Fig. 27. The case shown in the middle frame for the linearly

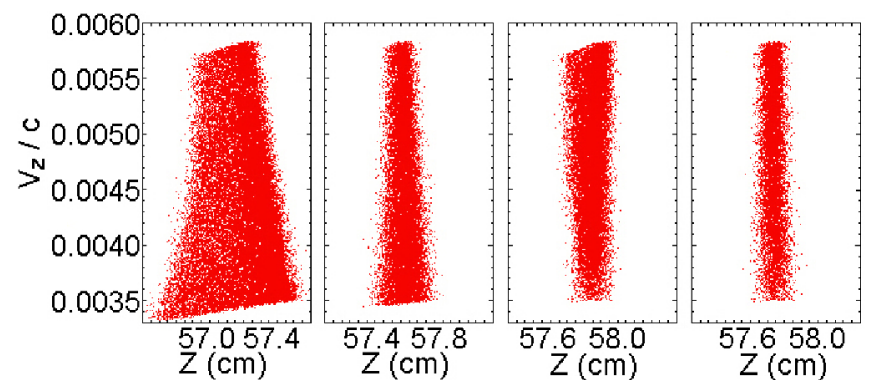

FIG. 26. (Color) $\left\{z, v_{z}\right\}$ beam phase space plots for the initially constant $r_{b}=2 \mathrm{~cm}$ (left), constant $r_{b}=1 \mathrm{~cm}$ (middle left), decreasing $J_{z}$ with linearly increasing $r_{b}$ from $r_{b}=0.1$ to $2 \mathrm{~cm}$ (middle right), and decreasing $J_{z}(t)$ with linearly increasing $r_{b}(t)$ from $r_{b}=0.1$ to $1 \mathrm{~cm}$ (right) cases near the simultaneous focal planes $z=+57.1 \mathrm{~cm}$ at $t=946.9 \mathrm{~ns}, z=+57.6 \mathrm{~cm}$ at $t=949.3 \mathrm{~ns}, \quad z=+57.85 \mathrm{~cm}$ at $t=951.0 \mathrm{~ns}, \quad$ and $z=$ $+57.85 \mathrm{~cm}$ at $t=950.7 \mathrm{~ns}$, respectively. 


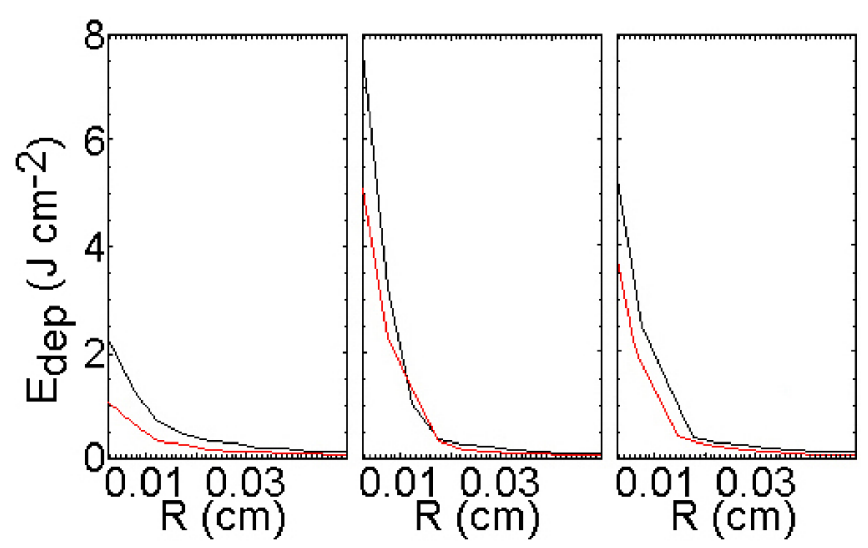

FIG. 27. (Color) Radial profiles of cumulative energy deposition $E_{\text {dep }}(r)$ using a $150 \mathrm{kG}$ solenoid and initially constant $J_{z}$ (left), decreasing $J_{z}(t)$ with linearly increasing $r_{b}(t)$ (middle), and halfdecreasing and half-increasing $J_{z}(t)$ with linearly half-increasing $r_{b}(t)$ and linearly half-decreasing $r_{b}(t)$ (right) profiles for the cases involving $r_{b}=1 \mathrm{~cm}$ (black) and $r_{b}=2 \mathrm{~cm}$ (red) at the focal planes given in Fig. 26.

increasing $r_{b}(t)$ from $r_{b}=0.1 \mathrm{~cm}$ to $r_{b}=1 \mathrm{~cm}$ recovers the original current compression ratio of 400 with a $t_{\mathrm{FWHM}}=0.6 \mathrm{~ns}$ and radius $(1 / e) r_{b}^{\text {foc }}=80 \mu \mathrm{m}$, yielding a peak on-axis $E_{\text {dep }}^{\text {peak }}=7.7 \mathrm{~J} \mathrm{~cm}^{-2}$ and approximate intensity $8 \mathrm{GW} \mathrm{cm}^{-2}$. The results for all of the cases are listed in Table VI in terms of the compression ratio, full-width, halfmaximum pulse length, and peak on-axis energy deposition $E_{\text {dep }}^{\text {peak }}$. The cases involving relatively smaller amounts of on-axis energy deposition have relatively larger proportions of it residing at larger radii, although the fact is difficult to illustrate on the linear scale of Fig. 27.

In summary, the $r_{b}(t)$ profile produced by the transverse defocusing effect is beneficial for reducing the simultaneous focusing aberration normally imposed on a beam with constant $r_{b}(t)$ entering a final-focus solenoid. Further improvements for achieving simultaneous focusing in the laboratory using a final-focus solenoid could use timedependent magnetic focusing, in tandem with the calculated defocusing effect, in order to ensure a beam with a linearly increasing $r_{b}(t)$ exits the gap, rather than the approximately triangular (or, parabolic) profile presently available. Alternatively, a change in gap conducting boundary geometry that decreases the $E_{r}$ imbalance early

TABLE VI. Compression dependence on initial $r_{b}(t)$ profile using a $150 \mathrm{kG}$ solenoid.

\begin{tabular}{lccc}
\hline \hline Initial $r_{b}(t)$ & $I_{b}^{\text {max }} / I_{0}$ & $t_{\mathrm{FWHM}}$ & $E_{\text {dep }}^{\text {peak }}$ \\
\hline $2 \mathrm{~cm}$, constant & 100 & $2.7 \mathrm{~ns}$ & $1.1 \mathrm{~J} \mathrm{~cm}^{-2}$ \\
$1 \mathrm{~cm}$, constant & 310 & $0.8 \mathrm{~ns}$ & $2.3 \mathrm{~J} \mathrm{~cm}^{-2}$ \\
$2 \mathrm{~cm}$, triangular & 275 & $0.8 \mathrm{~ns}$ & $3.8 \mathrm{~J} \mathrm{~cm}^{-2}$ \\
$1 \mathrm{~cm}$, triangular & 375 & $0.7 \mathrm{~ns}$ & $5.3 \mathrm{~J} \mathrm{~cm}^{-2}$ \\
$2 \mathrm{~cm}$, linear increase & 290 & $1.0 \mathrm{~ns}$ & $5.0 \mathrm{~J} \mathrm{~cm}^{-2}$ \\
$1 \mathrm{~cm}$, linear increase & 400 & $0.6 \mathrm{~ns}$ & $7.7 \mathrm{~J} \mathrm{~cm}^{-2}$ \\
\hline \hline
\end{tabular}

in time but exacerbates the defocusing late in time, can also result in a linearly increasing $r_{b}(t)$ profile.

\section{SUMMARY AND CONCLUSIONS}

In this paper, the derivation of an ideal velocity tilt, and the voltage waveform $V(t)$ that produces it, was presented for a cold ion beam encountering an infinitely thin acceleration gap and subsequently undergoing longitudinal compression (Sec. II). Under such idealized circumstances, no theoretical finite upper bound on the bunch compression exists. However, such a perfectly compressed beam cannot be realized in experiments, since all beams have finite longitudinal temperature $T_{\|}$, any acceleration gap must have finite spatial extent, and other realities must be considered. Ultimately, laboratory constraints and other physics limitations placed finite upper bounds on the achievable compression factors and minimum pulse lengths.

This paper employed PIC simulations in order to evaluate the neutralized drift compression of intense ion beams, and systematically studied the inclusion of various realistic effects in order to assess the upper limits of longitudinal compression in the laboratory compared to the ideal (Sec. III). Quantitative examples examined the dependence of longitudinal compression on many system parameters, such as the finite-size acceleration gap and voltage waveform (Sec. III A), initial longitudinal beam temperature $T_{\|}$ (Sec. III B), initial pulse length $t_{p}$ and intended fractional tilt $f$ (Sec. III C), and variations in initial beam kinetic energy $E_{0}$ and distribution (Sec. III D).

First, ideal and realistic types of acceleration gap and voltage waveform (the infinitely thin and $3 \mathrm{~cm}$ long gaps and sharp and smooth waveforms, respectively) were studied in order to ascertain the origin of various effects. The electric field encountered by the beam in the gap had significant extent in space and time, relative to the initial bunch length $L_{b}$ and $t_{p}$ of the beam, and gave rise to a number of complications. For example, the finite-size gap was shown to impart an effective longitudinal temperature to an otherwise cold beam due to the coupling of transverse and longitudinal dynamics. Also, the inclusion of the extra voltage in the smooth compared to the sharp waveform increased the relative $f$ and compression ratio by approximately $1 / 3$ with only a small increase in minimum pulse duration at focus.

Second, it was shown that the $T_{\|}$, whether present in the initial beam or imparted by the gap, contributed to longitudinal aberration of the focal plane, resulting in decreased amounts of compression, increased pulse lengths, and increased axial tolerance for diagnostic or target positioning. Theory and simulation revealed that slower head particles suffer from such focusing aberration more than faster tail particles. An analytical equation was derived for the minimum pulse length of an ideally tilted beam with finite $T_{\|}$, and accounted for the energy dependence of the longitudinal focusing aberration. The calculation agreed well with 
PIC simulation, when a constant accounted for the effective $45 \% T_{\|}$increase due to the smooth waveform and finite-size gap effects, for the parameters under consideration. The compression ratio and pulse length at focus were demonstrated to decrease and increase, respectively, with $\sqrt{T_{\|}}$dependence, which was the limiting physics on longitudinal compression using optimized waveforms and plentiful plasma supply. Beam temperatures $T_{\|}$consistent with measurements reduced the achievable compression ratios to $<500$ for NDCX-relevant parameters, and caused asymmetric current profiles away from the focal plane.

Third, the axial compression dependence on initial $t_{p}$ and intended $f$ was also investigated, since they comprised the two voltage waveform $V(t)$ parameters adjustable by the experimenter. The finite-size gap reduced the intended fractional tilt $f$ as a function of the initial $L_{b}$ and $t_{p}$ compared to the gap size and transit time, and should also be considered an effective $T_{\|}$increase. Shorter initial $t_{p}$ achieved reduced tilts from the gap effects, as the particle transit times became a significant fraction of $t_{p}$. A plateau effect in initial $t_{p}$ was shown to occur, where the longitudinal focusing aberration over longer drift lengths dominated the increases in compression, indicating the presence of a trade-off consideration between current compression and pulse duration. The dependence on intended $f$ was discussed and agreed well with theory. Larger tilts are generally desirable in experiments, but their realization is limited by the volt-second capability and hold-off requirements of the induction module, as well as concerns over increased transverse focusing aberration. A balance between longer initial $t_{p}$ and larger $f$ was discussed, since both increased the compression ratio, but had opposite effects on the minimum pulse length $t_{\mathrm{FWHM}}$, drift length $L_{d}$, and longitudinal focusing aberration. An optimum $L_{d}$ was argued to exist, for a given set of beam parameters, that balances the competing effects determining the compression ratios and minimum pulse lengths at focus.

Fourth, quantitative examples presented in this paper outlined the dependence of axial compression on initial kinetic energy, whether constant in time or not, and distribution variations. Since the slope of the waveform was only ideal for one particular $E_{0}$ and assumed a cold $T_{\|}=$ $0 \mathrm{eV}$ beam, simulations warned that small $( \pm 2.5-5 \%)$ constant differences and $( \pm 2 \%)$ temporal drifts in initial $E_{0}$ in the NDCX experiments, whether due to shot-to-shot variation, systematic uncertainty or inaccuracy, or hardware limitations, caused significant departure from the optimum compression parameters. It was also demonstrated that experimental beam energy scans to align the plane of the diagnostic with a peak compression result, while holding the waveform fixed, did not unambiguously determine the optimum compression, and a method for doing so was provided. Lastly, the beam's thermal distribution also affected the temporal shape of the current profiles and amount of longitudinal focusing aberration.
Beams with Waterbag distributions were shown to result in markedly asymmetric current profiles and reduced compression ratios, compared to the bell-shaped Maxwellian distribution results.

Future experiments seek to transversely focus a beam to a submillimeter spot coincident with the longitudinal focal plane using a strong final-focus solenoid (Sec. IV), in order to offset the radial defocusing effect inherent in the procedure of applying an axial velocity tilt (Sec. IVA). However, transverse and longitudinal focusing aberrations occurred due to the variations in beam radius and axial beam energies entering the solenoid (Sec. IV B). Simulations involving beams with various initial $r_{b}(t)$ and $J_{z}(t)$ showed the simultaneous compression dependence on the beam's phase space, due to the coupling of the transverse and longitudinal dynamics within the solenoid. The transverse defocusing effect pulse-shaped a uniform beam into one with a triangular (or, parabolic) $r_{b}(t)$ profile and was shown to be beneficial for compression, compared to the constant $r_{b}(t)$ case, for reducing simultaneous focusing aberration within the constant solenoidal $\mathbf{B}(r, z)$ fields.

In summary, no universally valid analytic model exists for determining the expected current compression ratio (and minimum pulse length) at focus because of the general intractability of including its dependence on many parameters, such as voltage waveform accuracy and finite-size acceleration gap geometry, beam parameters including the longitudinal temperature and distribution type, neutralization processes by the background plasma, and other deleterious effects. Therefore, PIC simulations were employed as the most self-consistent method for investigating many of the major realistic effects that contribute to the physical and technological limits of longitudinal compression in the laboratory.

Future publications in this area will evaluate experimental plasma source flow profiles for ion beam current density compression experiments, as well as address the cumulative effects of partial neutralization on charge bunch compression, such as increases to the beam's minimum radius, transverse and longitudinal emittance, and minimum pulse length at the simultaneous focal plane.

\section{ACKNOWLEDGMENTS}

This research was supported by the U.S. Department of Energy under the auspices of the Heavy Ion Fusion Science Virtual National Laboratory. The authors would like to acknowledge useful discussions with Dr. J.J. Barnard, Dr. A. Friedman, Dr. E. Henestroza, Dr. I. D. Kaganovich, Dr. P.A. Seidl, Dr. E. A. Startsev, and Dr. D. R. Welch.

[1] S. S. Yu, W. R. Meier, R. P. Abbott, J. J. Barnard, T. Brown, D. A. Callahan, C. Debonnel, P. Heitzenroeder, J.F. Latkowski, B. G. Logan, S. J. Pemberton, P. F. Peterson, 
D. V. Rose, G.-L. Sabbi, W. M. Sharp, and D. R. Welch, Fusion Sci. Technol. 44, 266 (2003).

[2] B. G. Logan, F. M. Bieniosek, C. M. Celata, E. Henestroza, J. W. Kwan, E. P. Lee, M. Leitner, P. K. Roy, P. A. Seidl, S. Eylon, J.-L. Vay, W. L. Waldron, S. S. Yu, J. J. Barnard, D. A. Callahan, R. H. Cohen, A. Friedman, D. P. Grote, M. Kireeff Covo, W. R. Meier, A. W. Molvik, S. M. Lund, R. C. Davidson, P.C. Efthimion, E.P. Gislon, L. R. Grisham, I. D. Kaganovich, H. Qin, E. A. Startsev, D. V. Rose, D. R. Welch, C. L. Olson, R. A. Kishek, P. O'Shea, I. Haber, and L. R. Prost, Nucl. Fusion 45, 131 (2005).

[3] B. Y. Sharkov, N. N. Alexeev, M. M. Basko, M.D. Churazov, D. G. Koshkarev, S. A. Medin, Y.N. Orlov, and V. M. Suslin, Nucl. Fusion 45, S291 (2005).

[4] T. Kikuchi, T. Someya, S. Kawata, M. Nakajima, K. Horioka, and T. Katayama, Nucl. Instrum. Methods Phys. Res., Sect. A 558, 122 (2006).

[5] D. A. Callahan, Fusion Eng. Des. 32, 441 (1996).

[6] B. G. Logan and D. A. Callahan, Nucl. Instrum. Methods Phys. Res., Sect. A 415, 468 (1998).

[7] A. B. Sefkow, R.C. Davidson, I. D. Kaganovich, E. P. Gilson, P. K. Roy, P. A. Seidl, S.S. Yu, D. R. Welch, D. V. Rose, and J. J. Barnard, Nucl. Instrum. Methods Phys. Res., Sect. A 577, 289 (2007).

[8] E. Henestroza, S. Eylon, P. K. Roy, S. S. Yu, A. Anders, F. M. Bieniosek, W. G. Greenway, B. G. Logan, R. A. MacGill, D. B. Shuman, D. L. Vanecek, W.L. Waldron, W. M. Sharp, T.L. Houck, R.C. Davidson, P.C. Efthimion, E. P. Gilson, A. B. Sefkow, D. R. Welch, D. V. Rose, and C.L. Olson, Phys. Rev. ST Accel. Beams 7, 083501 (2004).

[9] I. D. Kaganovich, G. Shvets, E. Startsev, and R.C. Davidson, Phys. Plasmas 8, 4180 (2001).

[10] D. V. Rose, D. R. Welch, B. V. Oliver, R. E. Clark, W. M. Sharp, and A. Friedman, Nucl. Instrum. Methods Phys. Res., Sect. A 464, 299 (2001).

[11] P. K. Roy, S. S. Yu, E. Henestroza, A. Anders, F. M. Bieniosek, J. Coleman, S. Eylon, W. G. Greenway, M. Leitner, B. G. Logan, W. L. Waldron, D. R. Welch, C. Thoma, A. B. Sefkow, E.P. Gilson, P.C. Efthimion, and R. C. Davidson, Phys. Rev. Lett. 95, 234801 (2005).

[12] N.C. Christofilos, R.E. Hester, W. A. S. Lamb, D. D. Reagan, W. A. Sherwood, and R.E. Wright, Rev. Sci.
Instrum. 35, 886 (1964).

[13] A. B. Sefkow, R. C. Davidson, P.C. Efthimion, E. P. Gilson, S.S. Yu, P.K. Roy, F. M. Bieniosek, J.E. Coleman, S. Eylon, W. G. Greenway, E. Henestroza, J. W. Kwan, D. L. Vanecek, W.L. Waldron, and D. R. Welch, Phys. Rev. ST Accel. Beams 9, 052801 (2006).

[14] A. B. Sefkow and R. C. Davidson, Phys. Rev. ST Accel. Beams 9, 090101 (2006).

[15] P. A. Seidl, J. Armijo, D. Baca, F. M. Bieniosek, J.E. Coleman, E. Henestroza, M. Leitner, B. G. Logan, P. K. Roy, W. L. Waldron, S.S. Yu, A. Friedman, M. KireeffCovo, D. Grote, A. W. Molvik, W. M. Sharp, J. L. Vay, I. Haber, A. B. Sefkow, E. P. Gilson, P. C. Efthimion, I. D. Kaganovich, R. C. Davidson, D. V. Rose, and D. R. Welch, Nucl. Instrum. Methods Phys. Res., Sect. A 577, 215 (2007).

[16] E. Henestroza (private communication).

[17] D. R. Welch, D. V. Rose, T. C. Genoni, S. S. Yu, and J. J. Barnard, Nucl. Instrum. Methods Phys. Res., Sect. A 544, 236 (2005).

[18] A. B. Sefkow, Ph.D. thesis, Princeton University, 2007.

[19] LSP is a software product of ATK Mission Research, Albuquerque, NM 87110.

[20] T. P. Hughes, S.S. Yu, and R. E. Clark, Phys. Rev. ST Accel. Beams 2, 110401 (1999).

[21] C. H. Thoma, D. R. Welch, S. Eylon, E. Henestroza, P. K. Roy, S.S. Yu, and E.P. Gilson, Proceedings of the 2005 Particle Accelerator Conference, 2005, p. 3765, http:// ieeexplore.ieee.org/xpls/abs_all.jsp?arnumber=1591698.

[22] M. Reiser, Theory and Design of Charged Particle Beams (John Wiley \& Sons, Inc., New York, 1994).

[23] R. C. Davidson and H. Qin, Phys. Rev. ST Accel. Beams 8, 064201 (2005).

[24] D. R. Welch, D. V. Rose, C. H. Thoma, A. B. Sefkow, I. D. Kaganovich, P. A. Seidl, S. S. Yu, and P. K. Roy, Nucl. Instrum. Methods Phys. Res., Sect. A 577, 231 (2007).

[25] D. R. Welch, D. V. Rose, S. S. Yu, and C. L. Olson, Proceedings of the 2003 Particle Accelerator Conference, 2003, p. 2685, http://ieeexplore.ieee.org/ xpls/abs_all.jsp?arnumber=1289230.

[26] C. H. Thoma, Mission Research Corporation, Report No. MRC/ABQ-R-2070, 2002, p. 1. 